\title{
The Essence of the Little Box Challenge-Part B: Hardware Demonstrators \& Comparative Evaluations
}

\author{
Dominik Neumayr, Dominik Bortis, and Johann Walter Kolar
}

\begin{abstract}
In order to expedite the development of power electronic systems towards higher power density and efficiency at a lower cost of implementation, Google and IEEE initiated the Google Little Box Challenge (GLBC) aiming for the worldwide smallest 2 kVA/450 VDC/230 VAC single-phase PV inverter with $\eta$ $>95 \%$ CEC weighted efficiency and an air-cooled case temperature of less than $60^{\circ} \mathrm{C}$ by using latest power semiconductor technology and innovative topological concepts. This paper, i.e. Part B of a discussion of The Essence of the Little Box Challenge, presents the hardware implementations and novel control concepts of two GaN-based inverter systems selected by the authors to counter the challenge: (i) Little Box 1.0 (LB 1.0), a H-bridge inverter with two interleaved bridge-legs both operated with Triangular Current Mode (TCM) modulation which features a power density of 8.18 $\mathrm{kW} / \mathrm{dm}^{3}\left(134 \mathrm{~W} / \mathrm{in}^{3}\right)$ and a nominal efficiency of $96.4 \%$ and (ii) Little Box 2.0 (LB 2.0), an inverter topology with single bridgeleg $\mathrm{DC} /|\mathrm{AC}|$ buck-stage operated with constant frequency PWM and a subsequent $|\mathrm{AC}| / \mathrm{AC} \mathrm{H}$-bridge unfolder, which features a remarkable power density of $14.8 \mathrm{~kW} / \mathrm{dm}^{3}\left(243 \mathrm{~W} / \mathrm{in}^{3}\right)$ and a nominal efficiency of $\mathbf{9 7 . 4 \%}$. Implemented using latest GaN power semiconductor technology, Zero Voltage Switching (ZVS) throughout the $\mathrm{AC}$ period and a variable switching frequency in the range of $200 \mathrm{kHz}^{-1} \mathrm{MHz}$ in order to shrink the size of filter passives, the LB 1.0 was ranked among the top 10 out of $100+$ teams actively participating in the GLBC. The LB 2.0 is the result of further research and considers lessons learned from the GLBC and achieves despite moderate $140 \mathrm{kHz}$ constant frequency PWM and hard-switching around the peak of the AC output current a higher power density $\rho$ and a higher efficiency $\eta$. For both implemented prototypes experimental results are provided to confirm that all GLBC technical requirements are met. The experimental results include steady-state and stepresponse waveforms, EMI and ground current measurements, as well as efficiency and operating temperature measurements. The reason for the $\eta \rho$-performance improvement of LB 2.0 over LB 1.0 are then discussed in detail. Furthermore, the solutions of other GLBC finalists are described and then compared to the performance achieved with the hardware prototypes presented in this paper. This leads to findings of general importance and provides key guideline for the future development of ultracompact power electronic converters.
\end{abstract}

Index Terms - GaN, high power density, little box challenge, microinverter, power pulsation buffer, PV inverter, WBG, zero voltage switching.

Manuscript received October 10, 2019; revised March 3, 2020; accepted April 1, 2020. Date of publication September 30, 2020; date of current version September 1, 2020. (Corresponding author: Dominik Neumayr.)

All authors are with Power Electronic Systems Laboratory, ETH Zurich, ETL I12, Physikstrasse 3, 8092 Zurich, Switzerland (e-mail: neumayr@lem.ee.ethz. ch).

Digital Object Identifier 10.24295/CPSSTPEA.2020.00022

\section{INTRODUCTION}

N Part A of the paper at hand [1], the key design challenges and 1 the technical concepts adopted by the authors to implement an ultra-compact single-phase inverter and overcome the Google Little Box Challenge (GLBC, [2]) were described in detail and are briefly summarized in the following. In order to drastically shrink the size of the converter bridge-legs and EMI filter passives and break through the status quo in power density, GaN wide bandgap (WBG) semiconductor technology was selected by the authors to enable a high switching frequency in the range of $100 \mathrm{kHz}^{-1} \mathrm{MHz}$ [3]. By means of the widely accepted Triangular Current Mode (TCM) control technique [4], [5], Zero Voltage Switching of a bridge-leg can be achieved in every operating point throughout the AC period which results in low switching losses despite the high switching frequencies but requires a Zero Crossing Detection (ZCD) circuitry and sophisticated FPGA control for a robust implementation. In contrast to TCM control, conventional PWM features a constant switching frequency but suffers from high turn-on switching losses which limits the maximal feasible switching frequency. However, this drawback is mitigated by the fact that with a relatively large current ripple, i.e. a design with small filter inductance value, also for PWM the average switching losses can be considerably reduced and a switching frequency of up to a few hundreds of $\mathrm{kHz}$ is feasible. As GaN semiconductor technology enables both softand hard-switching in a totem-pole bridge-leg configuration (as opposed to Si MOSFETs), both bridge-leg control strategies were considered for the implementation of the Google Little Box inverter. Furthermore, the authors selected a 2-level implementation of the bridge-legs over a multilevel implementation because of the anticipated increase in volume introduced by the higher semiconductor count, the increased gate driving requirement (supply voltage and gate signal isolation), and because of the more involved control system to facilitate capacitor balancing under all operating conditions. In order to reduce the size of the energy storage required to cope with the $120 \mathrm{~Hz}$ power pulsation intrinsic to single-phase DC/AC converter system, the advantage of replacing bulky electrolytic DC-link capacitors with an additional auxiliary converter and well utilized buffer capacitors was emphasized in Part A of the paper. Among several concepts presented in literature, the buck-type Parallel Current Injector (PCI) buffer as depicted in Fig. 1(a) and (b) (cf. Fig. 3 and 11) [6]-[9], was selected by the authors to cope with the pulsating AC power since (i) it features excellent capacitor utilization and 


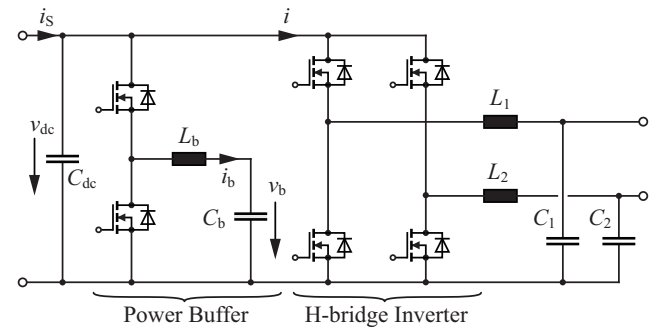

(a)

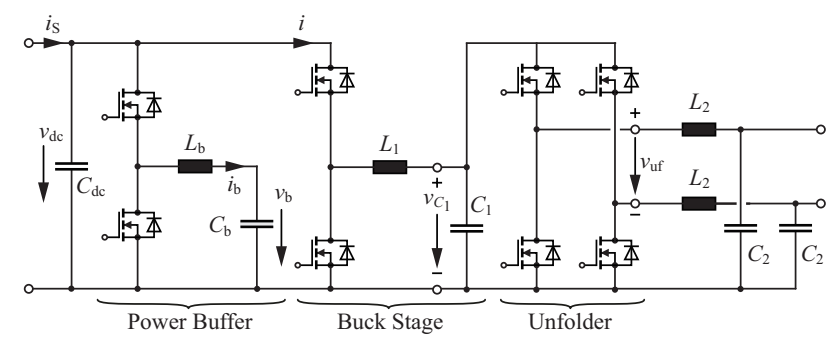

(b)

Fig. 1. (a) H-bridge inverter with DC-link referenced output filter and bucktype Parallel Current Injector (PCI) active power buffer (Little Box 1.0). (b) $\mathrm{DC} /|\mathrm{AC}|$ buck-stage and $\mid \mathrm{AC} / \mathrm{AC} \mathrm{H}$-bridge unfolder inverter with PCI active power buffer (Little Box 2.0).

(ii) allows to employ the same bridge-leg design as used in the main inverter to achieve maximal performance with minimal increase of overall complexity. As a consequence of the large feasible buffer voltage ripple, comparably small capacitance values in the range of $100-200 \mu \mathrm{F}$ are needed, thus realizing the buffer capacitor with ceramic capacitor technology becomes a viable option. Since the effective energy density of electrolytic capacitors is reduced due to lifetime related current stress constraints, $2.2 \mu \mathrm{F} / 450 \mathrm{~V}$ class II X6S MLCC and $2 \mu \mathrm{F} / 500 \mathrm{~V}$ CeraLink capacitors were identified to be the most promising candidates for realizing an ultra-compact power buffer and are adopted for the implementation of the buffer capacitor [10]-[12]. Regarding the specified ground current requirements of $50 \mathrm{~mA}$ (revised from initially specified $5 \mathrm{~mA}$ ), the difference between a 1 or 2 HF bridge-leg inverter design regarding the generation of a Low-Frequency (LF) Common-Mode (CM) output voltage component was analyzed and the merits of a DC-link referenced filter structure which allows a combined DM and CM filtering in a single stage was highlighted in Part A of the paper. Concerning the EMI requirements of the GLBC, the concept of 4D-interleaving [13] was introduced which allows to operate the interleaved bridgelegs with an optimal overlapping interval (with respect to the AC period) for maximal conversion efficiency while meeting the EMI requirements. Furthermore, to dissipate the generated power losses during operation of the inverter, a parallel-fin type heat sink with both ultra-flat blowers and conventional fans was considered by the authors for the optimal implementation of the forced-air cooling systems. It was identified that a heat sink using blowers performs best for comparably long cooling units offering a large baseplate area for direct component attachment, and a Because of the flat dimensions of the blower, a sandwich-like arrangement with two heat sinks at the top and

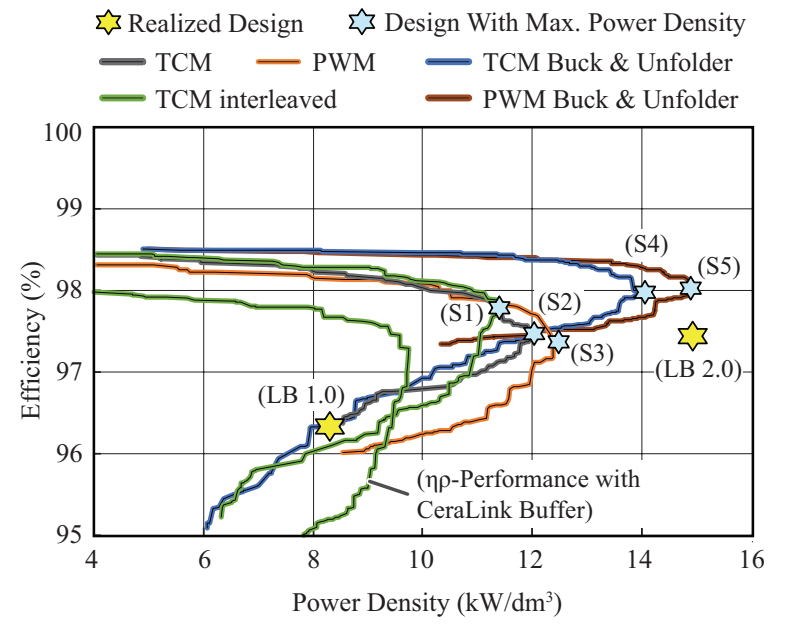

Fig. 2. Computed $\eta \rho$-performance of selected inverter concepts including the active power buffer for different operating modes (TCM/PWM) and with/ without interleaving of bridge-legs. Also indicated is the actual performance achieved with the implemented hardware prototypes as presented in this paper.

bottom and the converter in the center is possible (cf. Fig. 4). a heat sink design using fans performs best when the total length of the cooling unit is comparably short. Thus, this configuration is well suited for a component arrangement where only the power transistors are attached to the heat sink and the filter passives are cooled by the air flow exiting the heat sink (cf. Fig. 12).

Based on these key design considerations, two inverter concepts, (i) the H-bridge inverter with DC-link referenced output filter as shown in Fig. 1(a) (denominated as Little Box 1.0, LB 1.0, in the following) and (ii) the $\mathrm{DC} /|\mathrm{AC}|$ buck-stage and $\mid \mathrm{AC} / \mathrm{AC} \mathrm{H}$-bridge unfolder inverter topology as shown in Fig. 1(b) (denominated as Little Box 2.0, LB 2.0, in the following), both equipped with a buck-type PCI buffer to cope with the pulsating AC power, were selected for further analysis and a comparative evaluation. The results of a comprehensive multi-objective $\eta \rho$-Pareto optimization described in Part A (cf. Fig. 2 and Fig. 18(a) of Part A) indicate that, despite of higher switching losses, operation with constant switching frequency just below $150 \mathrm{kHz}$ PWM achieves a higher power density compared to TCM control. This is explained by the fact that, for the given GLBC specifications and the performance of the employed GaN semiconductor technology, the loss savings of operating with Zero Voltage Switching (ZVS) throughout the $\mathrm{AC}$ period are less compared to the added conduction losses caused by the high RMS current and remaining ZVS switching losses resulting from the TCM control. Furthermore, it is shown that with the $\mathrm{DC} /|\mathrm{AC}|$ buck-stage and $\mid \mathrm{AC} / \mathrm{AC} \mathrm{H}$-bridge unfolder inverter operated with PWM and a comparably large current ripple (small buck-stage filter inductance value) a power density of $14.7 \mathrm{~kW} / \mathrm{dm}^{3}\left(240 \mathrm{~W} / \mathrm{in}^{3}\right)$ with an efficiency of up to $98 \%$ at $2 \mathrm{~kW}$ output power is possible. Compared to the H-bridge inverter concept, this inverter therefore features a $\approx 15 \%-20 \%$ higher power density and a $1.7 \%$ higher efficiency at $2 \mathrm{~kW}$ rated power.

In this paper, i.e. Part B of a discussion of The Essence of 


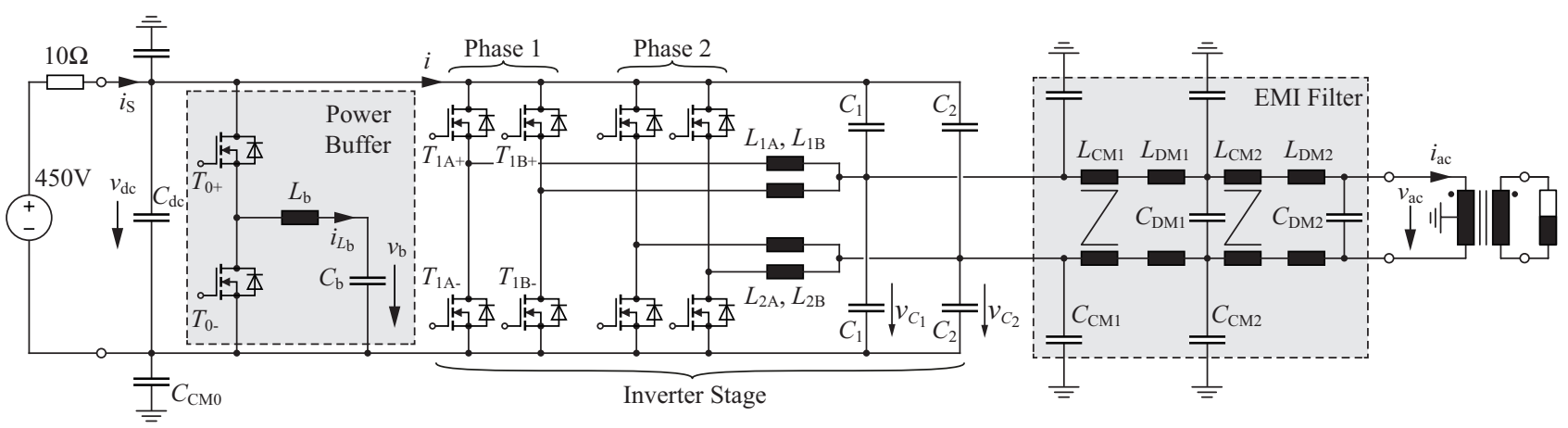

Fig. 3. Topology of the inverter denominated as Little Box 1.0 (LB 1.0) realized according to the Google Little Box Challenge (GLBC) technical specifications (cf. Table I in Part A) with the original $5 \mathrm{~mA}$ ground current limit. The systems consists of a H-bridge inverter with two interleaved bridge-legs per phase and a subsequent EMI output filter. The DC-side energy storage to compensate the fluctuating AC power is realized with a buck-type Parallel Current Injector (PCI) active power buffer.

the Little Box Challenge, the claimed $\eta \rho$-performance of the considered inverter concepts is confronted with the actually achieved performance figures of realized hardware prototypes (cf. LB 1.0 and LB 2.0 in Fig. 2). In Section II, the hardware prototype and results of experimental measurements on the LB 1.0, i.e. a 2-level H-bridge based inverter topology with interleaved bridge-legs, TCM operation with variable switching frequency in the range of $250-1000 \mathrm{kHz}$, and a buck-type PCI buffer employing CeraLink capacitor technology, are presented. The LB 1.0 was presented at the GLBC finals and, with a power density of $8.18 \mathrm{~kW} / \mathrm{dm}^{3}$ (134 $\mathrm{W} / \mathrm{in}^{3}$ ) and a nominal efficiency of $96.4 \%$, was ranked among the top 10 out of $100+$ contestants. In Section III, the hardware prototype and results of experimental measurements on the $\mathrm{LB}$ 2.0, i.e. a $\mathrm{DC} / \mathrm{AC} \mid$ buck-stage and $\mid \mathrm{AC} / \mathrm{AC}$ H-bridge unfolder inverter which considers all findings and lessons learned from the GLBC, are presented. Compared to the LB 1.0 presented at the GLBC finals, a further volume reduction of $40 \%$ is achieved while at the same time the power losses are reduced by almost $25 \%$. Subsequently, in Section IV the main reasons for the performance improvement of the LB 2.0 over the LB 1.0 are discussed in detail. Afterwards, the solutions of other GLBC finalist are briefly described and the claimed efficiency and power density is compared to the experimentally verified $\eta \rho$-performance of the inverter concept selected by the authors. Finally, Section V concludes this paper and summarized important findings which are providing key guidelines for the future power density improvements of industrial ultra-compact converter systems.

\section{Little Box 1.0 Demonstrator}

As mentioned in the introduction of this paper, a 2-level H-bridge topology was selected because ideally no low-fre-quency (LF) Common Mode (CM) voltage is generated at the output and thus the specified ground current limit can be met without the need for bulky CM chokes. As depicted in Fig. 3, each of the two output phases is implemented by means of two interleaved 2-level totem-pole bridge-legs and, to further reduce the size of the EMI filter, a DC-link referenced bridge-leg filter
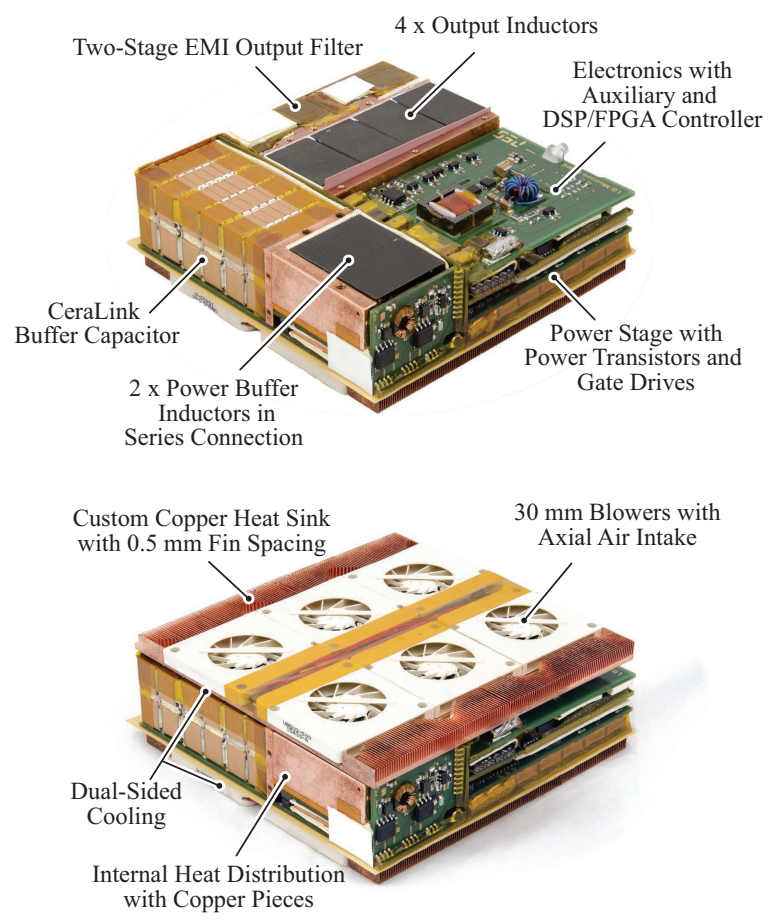

Fig. 4. Photograph of the realized LB 1.0 hardware (without housing) presented by the authors (ETH Zurich, PES), FH-IZM, and Fraza company, at the finals of the Google Little Box Challenge $(8.8 \mathrm{~cm} \times 8.9 \mathrm{~cm} \times 3 \mathrm{~cm}, 3.46$ in $\times 3.5$ in $\times 1.18$ in).

configuration is employed which facilitates a combined DM and CM filtering in a single stage. Furthermore, a buck-type PCI power buffer is employed to compensate the fluctuating $\mathrm{AC}$ power and mitigate the $\mathrm{DC}$-side voltage and current ripple to meet the specified requirements. In contrast to passive DClink buffering with electrolytic capacitors, the power buffer capacitor $C_{\mathrm{b}}$ can be cycled to a significant extent (large voltage ripple) and thus a much smaller capacitance value is required which translates into a reduced volume of the energy storage even though additional semiconductors, an additional filter inductor $L_{\mathrm{b}}$, and auxiliary circuits are needed. In Fig. 4, a photograph of the built LB 1.0 hardware prototype with a nominal efficiency of $96.4 \%$ and an overall volume of $240 \mathrm{~cm}^{3}$ 
resulting in a power density of $8.18 \mathrm{~kW} / \mathrm{dm}^{3}\left(134 \mathrm{~W} / \mathrm{in}^{3}\right)$ is shown. In the following, details of the implementation will be described along with the presentation of experimental results.

\section{A. Bridge-Leg Implementation}

For the implementation of the bridge-legs of the system, $600 \mathrm{~V} / 70 \mathrm{~m} \Omega$ normally-off Gallium Nitride (GaN) Gate Injection Transistors (CoolGaN, Samples from Infineon, [14]) are used in combination with a novel high-performance gate drive circuit [3], [15]. In order to reduce reverse conduction losses of the GaN transistors during the dead times, $600 \mathrm{~V} \mathrm{SiC}$ Schottky diodes from Wolfspeed are mounted in parallel to the power transistors. Each of the bridge-legs is operated with a TCM modulation scheme that enables zero voltage switching (ZVS) transitions in all operating points throughout an AC period [4], [5]. Since ZVS results in lower switching losses, the selected high (variable) switching frequency in the range of $200 \mathrm{kHz}-1 \mathrm{MHz}$ results in a small volume of the passive components, e.g. of the power stage filter inductors $L_{1 \mathrm{~A}}-L_{2 \mathrm{~B}}$. Furthermore, the high switching frequency in combination with the interleaving of two bridge-legs per output phase decreases the current ripple in the bridge-leg filter capacitors $C_{1 / 2}$ and doubles the effective switching frequency. Thus a higher cut-off frequency of the output filter is possible which in turn results in a reduced overall volume.

However, a high switching frequency also demands for suitable core materials and sophisticated inductor design in order to keep the high-frequency core and winding losses to a minimum. Addressing these challenges, the four output inductors of the inverter are realized based on a novel type of multiple-gap multiple parallel foil winding inductor [16]- [19]. Since the multiple small air gaps are evenly distributed over the full length of the inner core limb, the H-field in the winding window shows a quasi $1 \mathrm{D}$ distribution running in parallel to the inner limb. Consequently, a foil winding enabling a high filling factor can be used, since the H-field is aligned with the foil winding and thus ideally no eddy currents are induced. In order to counteract the skin effect at these high frequencies, the foil winding is realized with four parallel $20 \mu \mathrm{m}$ thin copper foils which are mutually isolated with a $7 \mu \mathrm{m}$ thin layer of Kapton. Furthermore, a sophisticated winding arrangement is used, which forces the current to flow evenly distributed in all four parallel copper layers, thus counteracting the proximity effect [16], [17]. The four output inductors of the inverter are realized based on this approach, where DMR51 MnZn Ferrite form DMEGC is used as high frequency core material. The inner limb of each output inductor has 24 air gaps surrounded by the four parallel copper foils with totally 16 turns, which gives an inductance value of around $10 \mu \mathrm{H}$. The inductor $L_{\mathrm{b}}=20 \mu \mathrm{H}$ of the power pulsation buffer was realized by connecting two 10 $\mu \mathrm{H}$ multi-gap inductors in series. Unfortunately, as analyzed in detail in [20] and discussed in Part A of this publication, the mechanical manufacturing and assembly of the thin ferrite plates resulted in excessive core losses in the inner limb which resulted in a relatively low quality factor of the implemented multi-gap inductors.
The capacitor of the PCI power buffer, $C_{\mathrm{b}}$, with an effective large-signal equivalent capacitance of $150 \mu \mathrm{F}$, was realized by means of 108 individual $2 \mu \mathrm{F} / 500 \mathrm{~V}$ CeraLink capacitors. By the courtesy of EPCOS/TDK, a custom package with 18 capacitor chips mounted together on silver coated copper lead frames was available which drastically simplified the construction of the buffer capacitor.

\section{B. Digital Control}

For the generation of the AC output voltage $v_{\mathrm{ac}}$, the two phase voltages are actively controlled to values symmetric around half of the DC-link voltage, i.e. $v_{C_{1}}=1 / 2\left(v_{\mathrm{ac}}+v_{\mathrm{dc}}\right)$ and $v_{C_{2}}=1 / 2\left(-v_{\mathrm{ac}}+v_{\mathrm{dc}}\right)$ (cf. Fig. 3). Since the sum of these phase voltages is constant, no LF CM output voltage is generated and hence LF ground currents are suppressed. The overall structure of the employed control system is shown in Fig. 5 and is composed of two subsystems dedicated to control the active power buffer and the inverter, respectively. To achieve a low THD and good transient performance, a cascaded voltage and current control feedback loop with Proportional-Resonant (PR) compensators [21] tuned at $60 \mathrm{~Hz}$ is used to regulate the $\mathrm{AC}$ output voltage. This control loop uses the AC quantities measured in the first EMI filter stage which is represented in a simplified manner by components $L_{3}$ and $C_{3}$ as shown in Fig. 5 . Based on the output of the AC voltage control loop,

$$
v_{C_{1-2}}^{*}=\bar{v}_{L_{3}}+v_{\mathrm{ac}}
$$

wherein $\bar{v}_{L_{3}}$ is the local average of the inductor voltage $v_{L_{3}}$, the reference value for the capacitor voltage of the two bridge-legs,

$$
\begin{gathered}
v_{C_{1}}^{*}=1 / 2\left(v_{C_{1-2}}^{*}+v_{\mathrm{dc}}\right), \\
v_{C_{2}}^{*}=1 / 2\left(-v_{C_{1-2}}^{*}+v_{\mathrm{dc}}\right),
\end{gathered}
$$

is derived. As can be seen, PI-compensators are used to control the voltages at $C_{1}$ and $C_{2}$. The reference current for $L_{1}$ and $L_{2}$ is then obtained by adding or subtracting the feed-forward term $i_{L_{3}}$ to the output of the PI-compensators, respectively. Based on the measurements of the DC-link voltage $v_{\mathrm{dc}}$ and the bridgeleg output voltages $v_{C_{1}}$ and $v_{C_{2}}$, circuit parameters such as the inductance value and the transistor output capacitances $\left(C_{\text {oss }}\right)$, a predictive current control algorithm calculates the turnon, turn-off and dead time interval of each bridge-leg such that the resulting inductor current features the characteristic triangular shape to achieve ZVS at both turn-on and turn-off of the bridge-leg while ensuring the correct average value $i_{L_{1 / 2}}^{*}$. To achieve a complete resonant transition at every switching instant throughout the AC period for a defined maximum dead time interval and limits imposed by the range of the variable switching frequency, a minimum constant reverse current of 5.0 A is used. The information of the inductor current ZeroCrossing (ZC) is used to update the remaining turn-on and turn-off interval to cope with measurement and parameter inaccuracies. To implement the digital control structure 


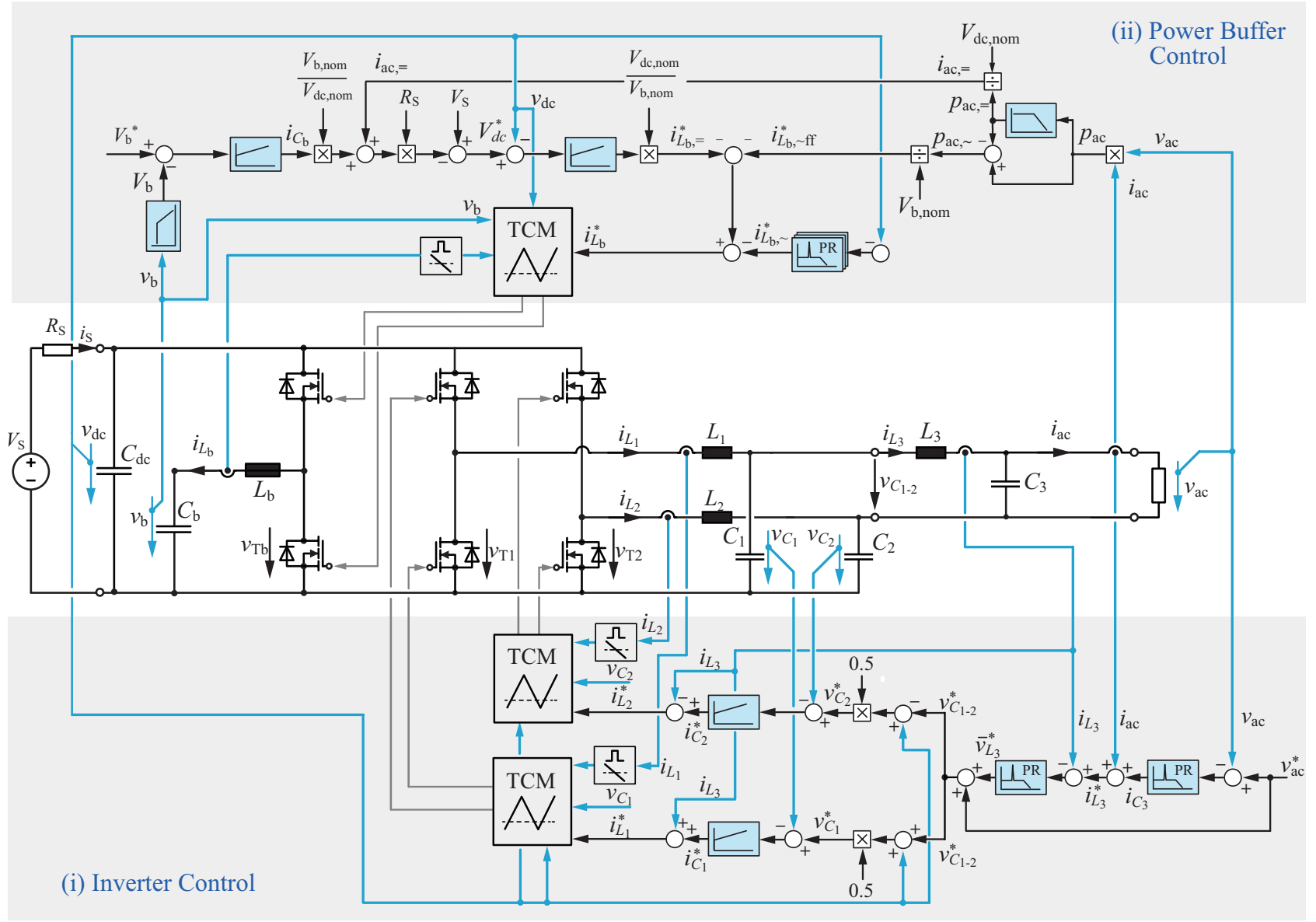

Fig. 5. Implemented digital control system of the Little Box 1.0 (LB 1.0). The inverter control structure is located below and the power buffer control structure is located above the power circuit diagram of the converter. For the sake of simplicity, the interleaved bridge-legs and the EMI filter are not included in the circuit diagram. The AC output voltage is controlled in the first DM filter stage which is represented by the components $L_{3}$ and $C_{3}$.

in hardware, a combination of a TI C2000 Delfino realtime microcontroller (TMS320F28335ZJZS) and a Lattice FPGA (LFXP2-5E-5MN132I) is used. The analog signal measurements, the feedback control system as depicted in Fig. 5, and the computation of the TCM timing intervals are implemented on the microcontroller and are executed with the fastest possible rate of $20 \mathrm{kHz}$. The timing intervals from the microcontroller and the current $\mathrm{ZC}$ signals are forwarded to the FPGA where the TCM modulator with a resolution of $5 \mathrm{~ns}$ (200 MHz) is implemented. Thus, for the given switching frequency range of $200 \mathrm{kHz}^{-1} \mathrm{MHz}$, the TCM timing variables from the microcontroller are updated only every $\approx 10-50$ switching cycles which justifies the twice per cycle occurring ZC feedback and adjustment of timing intervals. The TCM modulator also features the synchronization capability for the correct interleaving of the two bridge-legs for each output phase (cf. Fig. 3) in order to ensure the desired cancellation of the first harmonic in the current. The commands to activate and deactivate the individual bridge-legs depending on the position within the $\mathrm{AC}$ period and actual output power level (4D-interleaving, as described in Section III-C3 of Part A) are determined on the microcontroller and are then forwarded to the FPGA.
The selected magnetic saturation based Zero Crossing Detection (ZCD, cf. Fig. 5 of Part A, [22]) is implemented by means of a small toroidal core with an outer diameter of $4 \mathrm{~mm}(\mathrm{R} 4 \times 2.4 \mathrm{~mm} \times 1.6 \mathrm{~mm}, \mathrm{~B} 64290 \mathrm{P} 0036$ X830 from EPCOS). The selected core material is N30 which features a low saturation flux density and high permeability over a wide frequency range. The number of turns of the secondary (measurement) winding is set to $N_{\mathrm{s}}=10$. Depending on the inductor current slope $\left(\mathrm{d} i_{\mathrm{L}} / \mathrm{d} t\right)$, with this number of turns the induced voltage reaches values from $20 \mathrm{~V}$ up to $160 \mathrm{~V}$, which makes the ZCD circuit robust against electric disturbances. However, with the variability of the induced voltage also the time delay of the detection of the current crossings slightly changes. The induced voltage is tracked with a fast comparator circuit (TLV3501, 4.5 ns propagation delay) in order to keep the signal delay short and is then digitally filtered in the FPGA.

The control system of the buck-type PCI power buffer is also depicted in Fig. 5. The main objective of the power buffer control system is to exactly compensate the pulsating power caused by the single-phase $\mathrm{AC}$ load, such that only a constant power $P_{\mathrm{S}}=V_{\mathrm{S}} \cdot i_{\mathrm{S}}$ is drawn from the DC source. First, $p_{\text {ac, } \sim \text { is }}$ calculated by means of subtracting the average power $\overline{p_{\mathrm{ac}}}=p_{\mathrm{ac},=}$, from the instantaneous load power $p_{\mathrm{ac}}=v_{\mathrm{ac}} \cdot i_{\mathrm{ac}}$, as shown in 
the upper right corner of Fig. 5, with measured output voltage $v_{\mathrm{ac}}$ and current $i_{\mathrm{ac}}$ of the inverter. Dividing power $p_{\mathrm{ac}, \sim}$ by the nominal average buffer capacitor voltage $V_{\mathrm{b} \text {,nom }}$ yields the compensation current reference $i_{L_{\mathrm{b}}, \sim f f}^{*}$ which is used as a feedforward term. Besides neglecting the instantaneous power in $L_{\mathrm{b}}$ and the inverter's EMI filter, inaccuracies and delays in the signal acquisition and computation of the controller, the fluctuating AC power cannot be perfectly compensated by means of the feed-forward control. Therefore, in order to eliminate any possible remaining voltage ripple at the DC link, additional resonant compensators [21],

$$
C_{\mathrm{R}, \mathrm{m}}(s)=\frac{2 K_{\mathrm{i}, \mathrm{m}} s}{s^{2}+(m \cdot \omega)^{2}},
$$

are employed, wherein parameter $m \in 2,4,6$ sets the resonant frequency of the compensator to the first three even multiples of the mains frequency. As long as there is a spectral component with frequency $m \cdot \omega$ in the error signal $e_{\mathrm{m}}=0-v_{\mathrm{dc}}$ seen by the respective resonant compensator, the amplitude of the sinusoidal controller output will increase, requesting more current of that particular frequency to be injected into the DC link.

The cascaded control loop depicted in the upper left corner in Fig. 5 is employed to keep the mean value of the buffer capacitor voltage $\bar{v}_{\mathrm{b}}=V_{\mathrm{b}}$ at a chosen reference. If only control aspects are considered, then the reference voltage of the buffer capacitor $V_{\mathrm{b}}^{*}$ would be set to a voltage level

$$
V_{\mathrm{b}, \text { mid }}=V_{\mathrm{dc}, \text { nom }} / \sqrt{2}=282.8 \mathrm{~V}
$$

corresponding to half of the maximal stored energy. Maintaining the bias of the buffer capacitor at $V_{\mathrm{b} \text {,mid }}$ results in symmetrical energy margins, and a load step-up or step-down can be handled equally well. However, since (i) the prevailing capacitance density of the considered ceramic capacitors is strongly dependent on the DC bias and (ii) the amplitude of current $i_{\mathrm{b}}$ is inversely proportional to $v_{\mathrm{b}}$, a compromise between transient handling capability and $\eta \rho$-performance must be made. Therefore, in case of the realized PCI buffer of the LB 1.0 with CeraLink capacitors, the reference voltage $V_{\mathrm{b}}^{*}$ is set to $300 \mathrm{~V}$.

As a result of the current injected at the DC-link to compensate the fluctuation portion of the $\mathrm{AC}$ power, the buffer capacitor voltage features a distinctive voltage ripple at twice the mains frequency. In order to extract the mean buffer voltage $V_{\mathrm{b}}$, a low-pass filter, specifically a moving-average filter with window size of one $120 \mathrm{~Hz}$ period, is employed. A PI-compensator is used to compute the current $i_{C_{\mathrm{b}}}$ needed to charge or discharge the buffer capacitor to meet the reference value. The inner loop of the cascaded PCI buffer control structure depicted in Fig. 5 is required to tightly regulate the average DC-link voltage under all load conditions. It should be noted that this control loop also contributes to the cancellation of any remaining low frequency voltage ripple at the DC- link. However, the resonant compensators can be tuned more aggressively and are thus more effective in eliminating the LF voltage ripple.

The input current reference of the converter is given by

$$
\dot{i}_{\mathrm{S}}^{*}=i_{C_{\mathrm{b}}} \frac{V_{\mathrm{b}, \text { nom }}}{V_{\mathrm{dc}, \text { nom }}}+\frac{p_{\mathrm{ac},=}}{V_{\mathrm{dc}, \text { nom }}},
$$

which includes the current needed to adjust the buffer capacitor bias and the current needed to provide the real power $P_{\mathrm{S}}$ $=p_{\mathrm{ac},=}$ to the load. The current $i_{C_{\mathrm{b}}}$ computed by the outer PIcontroller, is referred to the DC link voltage level by means of the static scaling factor $\frac{V_{\text {b,nom }}}{V_{\text {denom }}}$. Since it is assumed that with ideal control there is no $120 \mathrm{~Hz}$ ripple present in the DClink voltage, low-pass filtering of $v_{\mathrm{dc}}$ is not necessary which increases the phase-margin of the inner loop of the cascade. Given the converter input current, the DC-link voltage reference is calculated with the equation

$$
\begin{aligned}
V_{\mathrm{dc}}^{*} & =V_{\mathrm{S}}-R_{\mathrm{S}} \cdot i_{\mathrm{S}}^{*} \\
& =V_{\mathrm{S}}-R_{\mathrm{S}} \cdot\left(i_{C_{\mathrm{b}}} \cdot \frac{V_{\mathrm{b}, \text { nom }}}{V_{\mathrm{dc}, \text { nom }}}+\frac{p_{\mathrm{ac},=}}{V_{\mathrm{dc}, \text { nom }}}\right) .
\end{aligned}
$$

It should be noted that instead of computing $V_{\mathrm{dc}}^{*}$ under the assumption of a constant input resistance $R_{\mathrm{S}}=10 \Omega$, an additional PI-compensator can be used to regulate the converter input current. The output of the PI-compensator is the required voltage across the input resistor $R_{\mathrm{s}}$ and is subtracted from the fixed source voltage $V_{\mathrm{S}}=450 \mathrm{~V}$ to obtain the operating point dependent DC-link voltage reference $V_{\mathrm{dc}}^{*}$.

The inner-loop PI-compensator then controls $v_{\mathrm{dc}}$ to meet the reference $V_{\mathrm{dc}}^{*}$. Referring the output of the PI-compensator to the buffer capacitor voltage level by means of scaling with $V_{\mathrm{dc}, \text { nom }} / V_{\mathrm{b} \text {,nom }}$ yields the mean buffer current reference $i_{L_{\mathrm{b}},=}^{*}$, required to keep both $V_{\mathrm{b}}$ and $v_{\mathrm{dc}}$ at the desired average values. Due to the cascaded structure, controlling the DC-link voltage has always priority over the mean buffer capacitor voltage. This has significant advantages in case of abrupt load changes, since the average buffer capacitor voltage $V_{\mathrm{b}}$ can be temporarily deflected from the reference $V_{\mathrm{b}}^{*}$, keeping $v_{\mathrm{dc}}$ tightly controlled. Assuming a strict unidirectional power flow from the DC supply (no current sinking capability), then, e.g. in case of an stepwise load drop from $1 \mathrm{~kW}$ to $0 \mathrm{~kW}$, the energy stored in the converter system at the very moment of the load change, namely the energy stored in the passive components of the inverter stage and the PCI buffer inductor, is absorbed in the larger buffer capacitor and not in the at least a factor of 10 smaller DC-link capacitance. This prevents critical overshoots or sags in $v_{\mathrm{DC}}$, even under harsh load transients. Eventually, the individual current references $i_{L_{\mathrm{b}},=}^{*}, i_{L_{\mathrm{b}}, \mathrm{ff}}^{*}$ and $i_{L_{\mathrm{b}}, * *}$ are combined to obtain a single reference $i_{\mathrm{LB}}^{*}$ of the PCI filter inductor current. Note the negative gain because of the imposed counting direction of $i_{L_{\mathrm{b}}}$. 


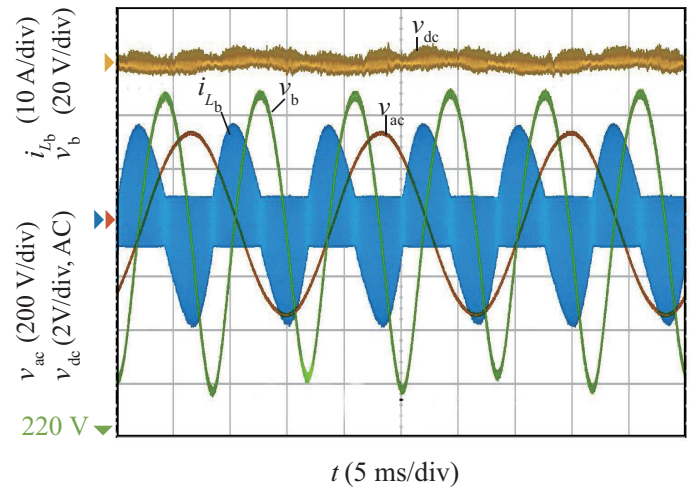

(a)

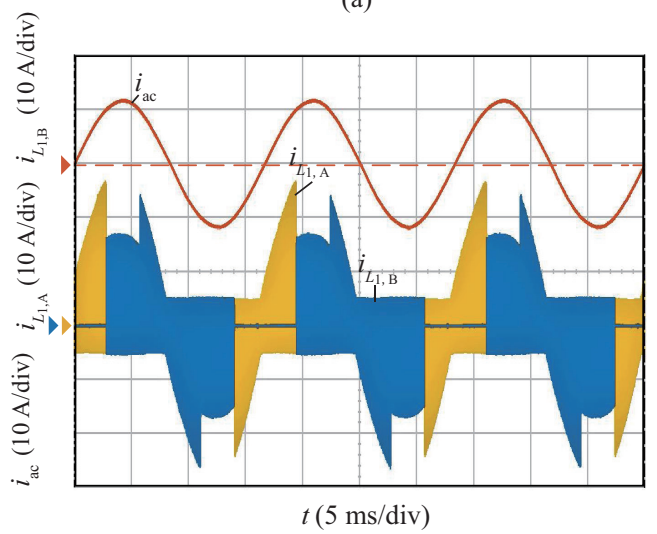

(b)

Fig. 6. Measured steady-state waveforms of the Little Box 1.0 (LB 1.0) prototype operated at the rated $2 \mathrm{~kW}$ output power. (a) The active power buffer eliminates the $120 \mathrm{~Hz}$ voltage ripple of the DC-link voltage $v_{\mathrm{dc}}$. The buffer voltage swing at rated power is designed to be around $110 \mathrm{~V}$. (b) Filtered output current and corresponding high-frequency Triangular Current Mode (TCM) modulated current $i_{L_{1, \mathrm{~A}}}$ and $i_{L_{1, \mathrm{~B}}}$ in the power stage inductors $L_{1, \mathrm{~A}}, L_{1, \mathrm{~B}}$ with 4D-interleaving (cf. Section III-C3 of Part A). The measurements were performed at unity power factor $\left(\cos \varphi_{0}=1\right)$ but the waveforms depicted in (a) and (b) were not captured simultaneously which explains the phase-shift between $v_{\mathrm{ac}}$ and $i_{\mathrm{ac}}$.

Likewise to the inverter, a predictive current control algorithm computes the correct timing intervals and a dedicated TCM modulator computes the high resolution bridge-leg control signals.

\section{Experimental Waveforms}

Fig. 6 shows the measured characteristic waveforms of the LB 1.0 prototype at steady-state and rated output power. Considering $v_{\mathrm{dc}}$ (yellow trace) in Fig. 6(a), it can be seen that the PCI power buffer effectively compensates the fluctuating AC power and successfully mitigates the $120 \mathrm{~Hz}$ voltage ripple. The resulting voltage swing present in the buffer capacitor voltage $v_{\mathrm{b}}$, amounts to $110 \mathrm{~V}$ and is superimposed to the bias or offset voltage $V_{\mathrm{b}}=300 \mathrm{~V}$. The characteristic TCM shape of the PCI buffer inductor current is also shown in Fig. 6(a). Fig. 6(b) displays the sinusoidal AC output current and the current in the inductor $L_{1, \mathrm{~A}}$ and $L_{1, \mathrm{~B}}$ of the two interleaved bridgelegs of output phase 1. Clearly visible is the outcome of the implemented 4D-interleaving, where both bridge-legs are only

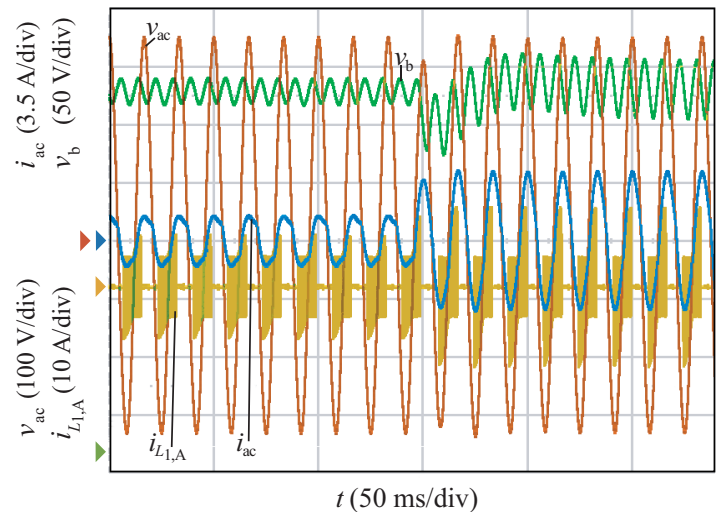

(a)

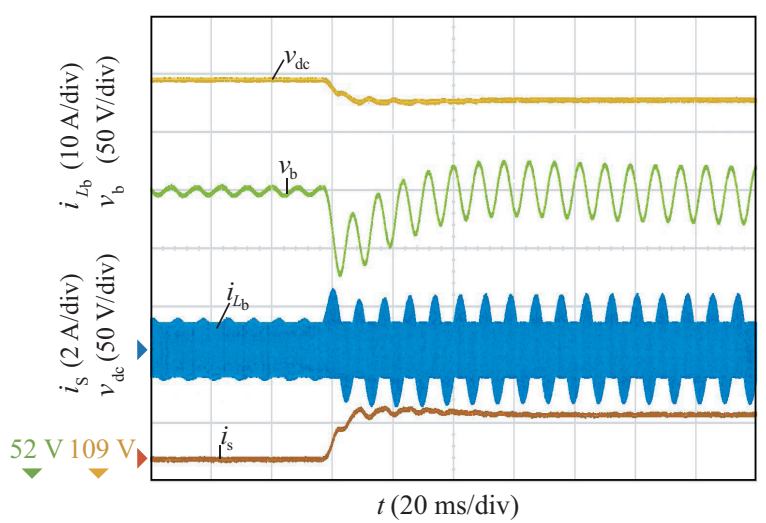

(b)

Fig. 7. Transient waveforms of the Little Box 1.0 (LB 1.0) subject to a stepwise increase in output power. (a) Measured AC output waveforms $v_{\mathrm{ac}}$ and $i_{\mathrm{ac}}$, and filter inductor current $i_{L_{1, \mathrm{~A}}}$ subject to a stepwise load increase from 230 $\mathrm{W}$ to $700 \mathrm{~W}$. (b) Measured DC-side and power buffer waveforms subject to a stepwise load increase from $0 \mathrm{~W}$ to $700 \mathrm{~W}$. The small ripple present in the buffer capacitor voltage prior to the load step despite $0 \mathrm{~W}$ of output power is due to the reactive power drawn by the EMI filter (DM capacitors). Note that the waveforms depicted in (a) and (b) were not captured simultaneously and exhibit a different time scale.

operated simultaneously at high instantaneous output power, i.e. around the peak value of $i_{\mathrm{ac}}$ in case of ohmic loads. The total overlap duration of the interleaved bridge-legs as shown in Fig. 6(b) amounts to $158^{\circ}$. The actual overlap duration in every operating point was determined empirically with the objective to increase the overall conversion efficiency while complying with the specified EMI regulations. Fig. 7 shows the measured waveforms of the LB 1.0 converter during a transient caused by a stepwise increase in output power. Fig. 7(a) shows the AC output waveforms during a load step from $230 \mathrm{~W}$ to 700 W. It can be seen that the transient is settled in less than $50 \mathrm{~ms}$ which clearly complies with the specified settling time of $1 \mathrm{~s}$ for a maximum specified load step of $500 \mathrm{~W}$. Fig. 7(b) shows the PCI buffer and DC-side related waveforms for a load step from $0 \mathrm{~W}$ to $700 \mathrm{~W}$. Triggered by the step, the average buffer capacitor voltage drops $50 \mathrm{~V}$ below the $300 \mathrm{~V}$ at steady-state. Simultaneously, the PCI buffer controller starts to compensate the power pulsation by means of injecting an appropriate 
current into the DC-link. As a consequence, a distinct $120 \mathrm{~Hz}$ voltage ripple develops at the buffer capacitor immediately after the load step. After a transient time of $60 \mathrm{~ms}$, the average buffer capacitor voltage has recovered and the fluctuating $\mathrm{AC}$ power is completely compensated by the active power buffer. Take note that the PCI is achieving a smooth transition of the DC-link voltage level which settles at a lower value after the transient due to the inserted $10 \Omega$ resistor $R_{\mathrm{s}}$ between the 450 V DC source and the DC-link (cf. Fig. 3). The reactive power drawn by the EMI filter (DM capacitors) of the inverter stage is compensated by the power buffer, thus a small ripple is present in the buffer capacitor voltage prior to the load step although no load is connected to the inverter.

\section{EMI Compliance}

In order to achieve a high filter attenuation while still keeping the filter components small, for the given circuit structure and selected frequency range a two-stage EMI output filter topology is employed as shown in Fig. 3. As can be noticed, although with the H-bridge topology ideally no LF CM voltage is generated at the inverter output, the CM inductors $L_{\mathrm{CM} 1}$ and $L_{\mathrm{CM} 2}$ as well as the CM capacitors $C_{\mathrm{CM} 1}$ and $C_{\mathrm{CM} 2}$ are still needed to filter the remaining $\mathrm{HF} \mathrm{CM}$ components. Note, that the bridge-legs of output phases 1 and 2 cannot be synchronized because of the TCM control and thus the $\mathrm{CM}$ noise elimination known from the H-bridge topology with bipolar PWM does not apply. In the given filter configuration, the output capacitors $C_{1}$ and $C_{2}$ not only help to attenuate the Differential Mode (DM) noise but also the CM noise, which means that if $C_{1}$ and $C_{2}$ are increased, the needed $\mathrm{CM}$ inductance can be decreased. Furthermore, since $C_{1 / 2}$ are either connected to the positive or negative DC-rail and thus no ground currents are generated, $C_{1 / 2}$ can be designed in the $\mu \mathrm{F}$-range which is much larger than the $\mathrm{CM}$ capacitor values of $C_{\mathrm{CM} 1}$ and $C_{\mathrm{CM} 2}$ which are more in the tens of $n F$-range. The only limiting factor for the capacitance of $C_{1}$, and also for the other DM capacitors $C_{\mathrm{DM} 1}$ and $C_{\mathrm{DM} 2}$, is the additional reactive power demand that causes larger currents and higher losses in the whole system. For the built prototype each $C_{1}$ is realized with four parallel and $C_{\mathrm{DM} 1}$ and $C_{\mathrm{DM} 2}$ with three parallel 2.2 $\mu \mathrm{F}, 450 \mathrm{~V}$ class II/X6S Multi-Layer Ceramic Capacitors (MLCC, C5750X6S2W225M250KA from EPCOS/TDK), since ceramic capacitors feature a much higher capacitance per unit volume than the conventionally used film capacitors. On the contrary, the dielectric material of the class II/X6S MLCCs exhibits substantial power loss when excited with a large-voltage swing at low frequencies (cf. Section II-B1 and Appendix C of Part A). Based on experimental measurements, the dissipated power per volume of the class II/X6S dielectric material is in the order of $4.7 \mathrm{~W} / \mathrm{cm}^{3}$ for an AC excitation with $2 \times \sqrt{2} \times 240 \mathrm{~V}=678.8 \mathrm{~V}$ peak-to-peak voltage at $60 \mathrm{~Hz}$

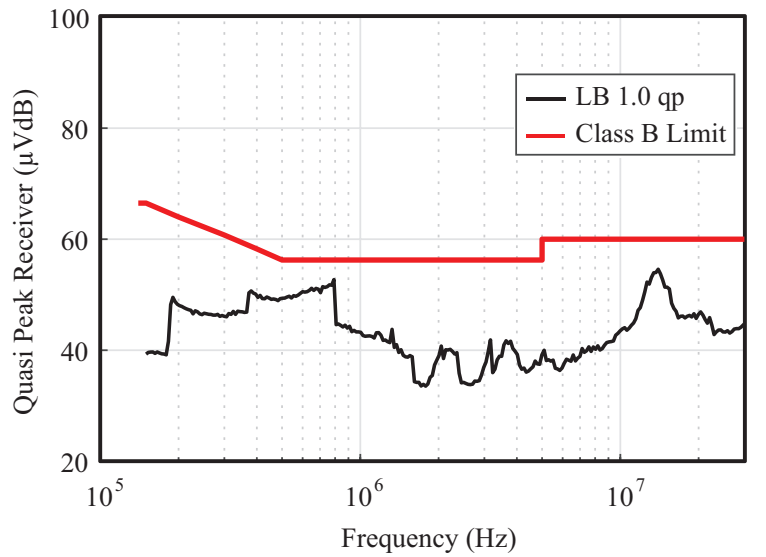

(a)

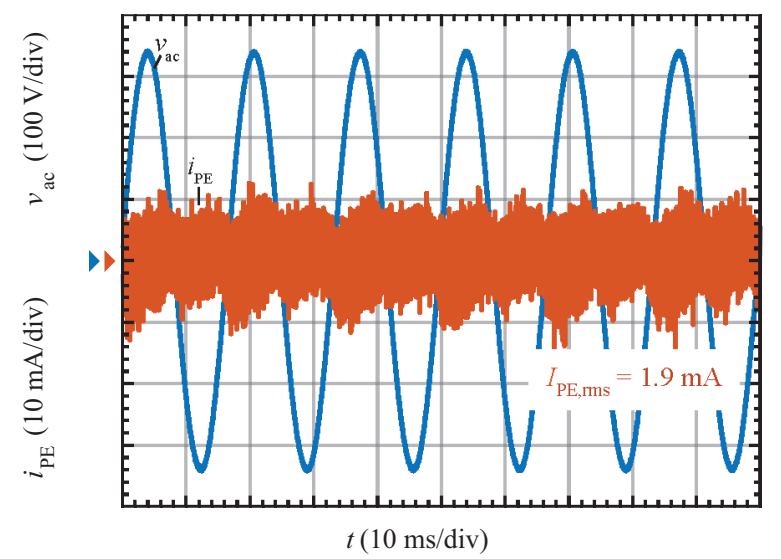

(b)

Fig. 8. (a) EMI noise spectrum of Little Box 1.0 (LB 1.0) at $2 \mathrm{~kW}$ output power measured with R\&S ENV216 single-phase LISN and R\&S ESPI7 test receiver. (b) Ground current of the LB 1.0 measured by means of inserting both $\mathrm{AC}$ output wires simultaneously into the clamp of a current probe (Teledyne CP030).

excitation frequency. Considering the voltage-and temperaturedependent capacitance of the selected components, the effective capacitance drops to approximately $650 \mathrm{nF}$ per piece which results in an additional reactive output filter power of around 200 VAr. The CM inductors $L_{\mathrm{CM} 1}$ and $L_{\mathrm{CM} 2}$ are built with toroidal cores from Vacuumschmelze which are based on the core material VITROPERM 500F that offers a high permeability and high saturation flux density (core type: T60006-L2012-W498, winding: 11 turns, $1 \mathrm{~mm}-\varnothing$ ). Even if the leakage inductance of the CM inductors contributes to the $\mathrm{DM}$ inductances, separate $\mathrm{DM}$ inductors, $L_{\mathrm{DM} 1}$ and $L_{\mathrm{DM} 2}$ are added to achieve the required DM attenuation. For all DM inductors the commercially available $10 \mu \mathrm{H}$-inductors from Coilcraft (XAL1010-103MED) are used. The CM capacitors $C_{\mathrm{CM} 0}, C_{\mathrm{CM} 1}$ and $C_{\mathrm{CM} 2}$ are implemented with $3 \times 100 \mathrm{nF}, 630 \mathrm{~V} /$ X7R MLCCs from TDK (C3225X7T2J104K160AC).

The quasi-peak EMI spectrum of the LB 1.0 hardware prototype with aluminium enclosure at $2 \mathrm{~kW}$ output power is depicted in Fig. 8(a). The results where obtained with the R\&S ESPI7 test receiver and the R\&S ENV216 single-phase 


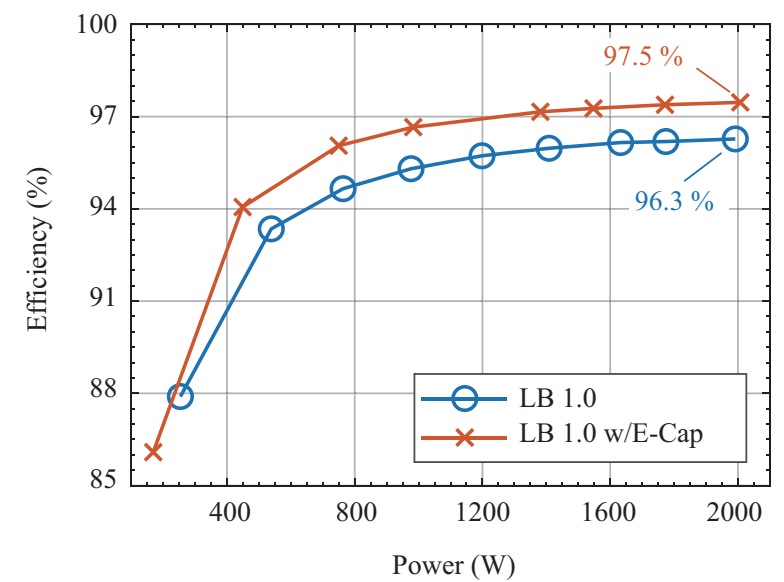

Fig. 9. Conversion efficiency of the Little Box 1.0 (LB 1.0) with respect to output power measured with a Yokogawa WT3000 precision power analyzer. The red curve with cross markers represents the measured efficiency of the inverter using passive DC-link buffering with electrolytic capacitors instead of the active power buffer.

LISN which was installed between the AC output of the converter and the resistive load bank. It can be clearly seen that the FCC Part 15 B conducted EMI limits are met. Due to the implemented TCM control with resulting variable switching frequency, the noise spectrum does not exhibit distinct peaks but instead is spread out over a wide frequency range.

In a preceding calibration test, the DC output terminals of the employed laboratory voltage source were connected directly to the resistive load bank. In a characteristic operating point, that is $450 \mathrm{~V} \mathrm{DC}$ and $5 \mathrm{~A}$, around $7.4 \mathrm{~mA}$ of ground current was measured by means of inserting both wires in the clamp of a Teledyne CP030 current probe. If the chassis of the supply was, in addition to the always persistent ground connection through the AC supply cable, also connected directly to the local ground/PE terminals of the EMI test setup, approximately $30 \mathrm{~mA}$ of ground current were detected. For this reason, a $\mathrm{CM}$ choke of $\approx 2 \mathrm{mH} @ 10 \mathrm{kHz}$ of nominal inductance, implemented with 5 turns around a toroidal VITROPERM 500F core (T60006-L2045-V102), was inserted between the DC supply (Sorensen SGI 600/17) and the converter input. Keep note that because of the low-impedance path provided by the $\mathrm{CM}$ capacitor $C_{\mathrm{CM} 0}$ at the input of the converter (cf. Fig. 3), a fair assessment of the ground current caused by the device under test is still possible.

The recorded ground current in the nominal operating point is depicted in Fig. 8(b) and was measured by inserting both AC output wires simultaneously into the clamp of the current probe(Teledyne CP030). The ground wire of the converter was connected to the local ground/PE terminal of the EMI test setup. The RMS value of the ground current amounts to only $1.9 \mathrm{~mA}$ and confirms the effectiveness of the designed CM filter and the symmetrical H-bridge inverter topology. It should be emphasized, that the LB 1.0 converter therefore complies to the original more stringent $5 \mathrm{~mA}$ ground current limit of the GLBC

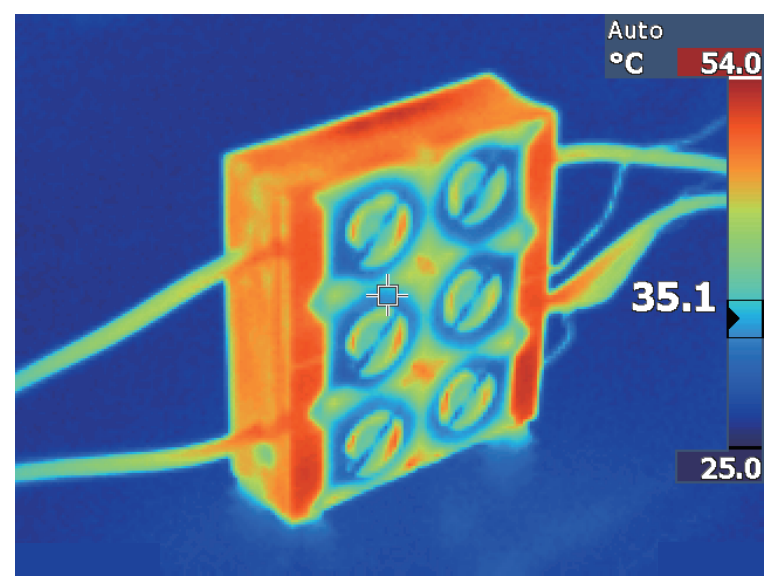

Fig. 10. Thermography image of the Little Box 1.0 (with housing) recorded with a FLUKE Ti10 infrared camera showing the steady-state temperature distribution at rated $2 \mathrm{~kW}$ output power.

which was later relaxed to $50 \mathrm{~mA}$ (cf. Section II of Part A).

\section{E. Efficiency}

The measured efficiency of the LB 1.0 prototype as a function of output power is depicted in Fig. 9. As indicated by the blue line with circle markers, the efficiency of the system with active power buffer exhibits a peak efficiency of $96.3 \%$ at rated output power. The corresponding CEC weighted efficiency amounts to $95.07 \%$, meeting the minimum efficiency requirements $(95 \%)$ of the GLBC. The red line with cross markers represents the measured efficiency of only the inverter with passive DC-link buffering by means of high-density electrolytic capacitors $(3 \times 493 \mu \mathrm{F} / 450 \mathrm{~V}, \mathrm{~B} 43991-\mathrm{X} 0009-\mathrm{A} 223$ from EPCOS/TDK). The peak efficiency amounts to $97.5 \%$ and the resulting CEC efficiency amounts to $96.3 \%$. The substantial reduction in efficiency by the operation of the PCI can be ascribed to the additional HF bridge-leg with multi-gap filter inductor and CeraLink buffer capacitor. In fact, as will be further discussed in Section IV, roughly $60 \%$ of the PCI power losses are caused by the CeraLink buffer capacitor.

\section{F. Cooling and Operating Temperature}

A dual-sided cooling arrangement(cf. Fig. 4) with ultra-flat custom-machined heat sink elements is used to extract the 74 $\mathrm{W}$ of power loss in the nominal operating point. The heat sink has a height of only $4.5 \mathrm{~mm}$ and employs 6 Sunon 5V DC micro blowers $(30 \times 30 \times 3 \mathrm{~mm})$ per element. The effective cooling system performance index (CSPI) amounts to $25 \mathrm{~W} /$ $\left(\mathrm{K} \mathrm{dm}^{3}\right)$ and also considers the heat distribution elements (thin copper pieces within the converter) needed to conduct the heat from the lossy components, e.g. the power inductors, to the baseplate of the respective heat sink. The thermography image in Fig. 10, recorded with a FLUKE Ti10 infrared camera, shows the steady-state temperature distribution of the LB 1.0 at $2 \mathrm{~kW}$ output power. As it can be clearly seen, the implemented cooling system is sufficient to meet the maximum allowed 


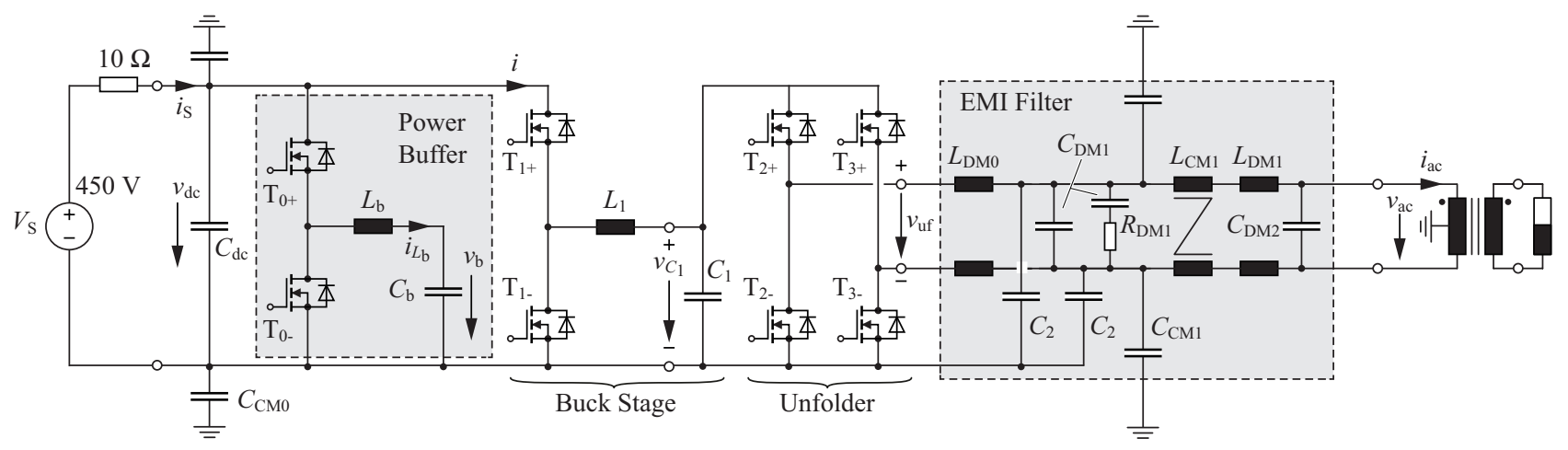

Fig. 11. Topology of the 2nd inverter realized according to the Google Little Box Challenge (GLBC) technical specifications with revised $50 \mathrm{~mA}$ earth current limit (Little Box 2.0, LB 2.0). The inverter is comprised of a DC/|AC| buck-stage with $\mid \mathrm{AC} / / \mathrm{AC}$ H-bridge unfolder and a subsequent EMI output filter. The DC-side energy storage to compensate the fluctuating AC power is also implemented with a buck-type Parallel Current Injector (PCI) active power buffer.

surface temperature requirement of $60^{\circ} \mathrm{C}$.

\section{LitTLE Box 2.0 DemONSTRATOR}

The inverter topology of the Little Box 2.0 (LB 2.0) as shown in Fig. 11 is based on a conventional $\mathrm{DC} /|\mathrm{AC}|$ buck converter which generates a rectified sinusoidal voltage $v_{\mathrm{Cl}}=$ $\left|\hat{V}_{\text {ac }} \cdot \sin (\omega t)\right|$ with respect to the negative DC-link rail and a subsequent $\mid \mathrm{AC} / / \mathrm{AC} \mathrm{H}$-bridge unfolder which generates the actually desired sinusoidal output voltage $v_{\text {uf }}$ [23]. Likewise to the LB 1.0 system, the DC-side energy storage to compensate the fluctuating $\mathrm{AC}$ power is realized with a buck-type PCI power buffer. Compared to the H-bridge topology of the LB 1.0 (cf. Fig. 3), the major advantage of this topology is that one half of the previously described inverter stage including bridge-leg filter inductors and capacitors can be completely omitted, thus volume and losses are saved and the system complexity is reduced. However, a higher ground current is to be expected since a LF CM voltage with a peak voltage equal to half the AC amplitude, i.e. $v_{\mathrm{CM}}=\hat{V}_{\mathrm{ac}} / 2 \cdot \sin (\omega t)$, is generated at the output(cf. Fig. 10(b) of Part A). The H-bridge unfolder was selected because, unlike the half-bridge unfolder(totempole inverter) [1], high $\mathrm{d} v / \mathrm{d} t$ transitions in the LF CM voltage are prevented and it is furthermore possible to utilize the DClink referenced filter configuration and therefore reduce the size of the EMI filter. In this regard, the $\mathrm{DC} /|\mathrm{AC}|$ buck-stage and $\mid \mathrm{AC} / \mathrm{AC} \mathrm{H}$-bridge unfolder concept became truly viable once the specified ground current limit was relaxed from 5 $\mathrm{mA}$ to $50 \mathrm{~mA}$ because of the expected volume reduction of the $\mathrm{CM}$ chokes due to the comparably high permissible total $\mathrm{CM}$ capacitance of $\approx \frac{50 \mathrm{~mA}}{2 \pi 60 \mathrm{~Hz} \cdot 1 / 2 \cdot 240 \mathrm{~V}}=1.1 \mu \mathrm{F}$.

In Fig. 12, a picture of the built LB 2.0 hardware prototype with an overall boxed volume of $135 \mathrm{~cm}^{3}$ (without housing) resulting in a remarkable power density of $14.8 \mathrm{~kW} / \mathrm{dm}^{3}(243$ $\mathrm{W} / \mathrm{in}^{3}$ ) and a nominal efficiency of $97.4 \%$ is shown. In the following, details of the implementation will be described along with the presentation of experimental results.

\section{A. Bridge-Leg Implementation}

For the implementation of the PCI and inverter buck-stage
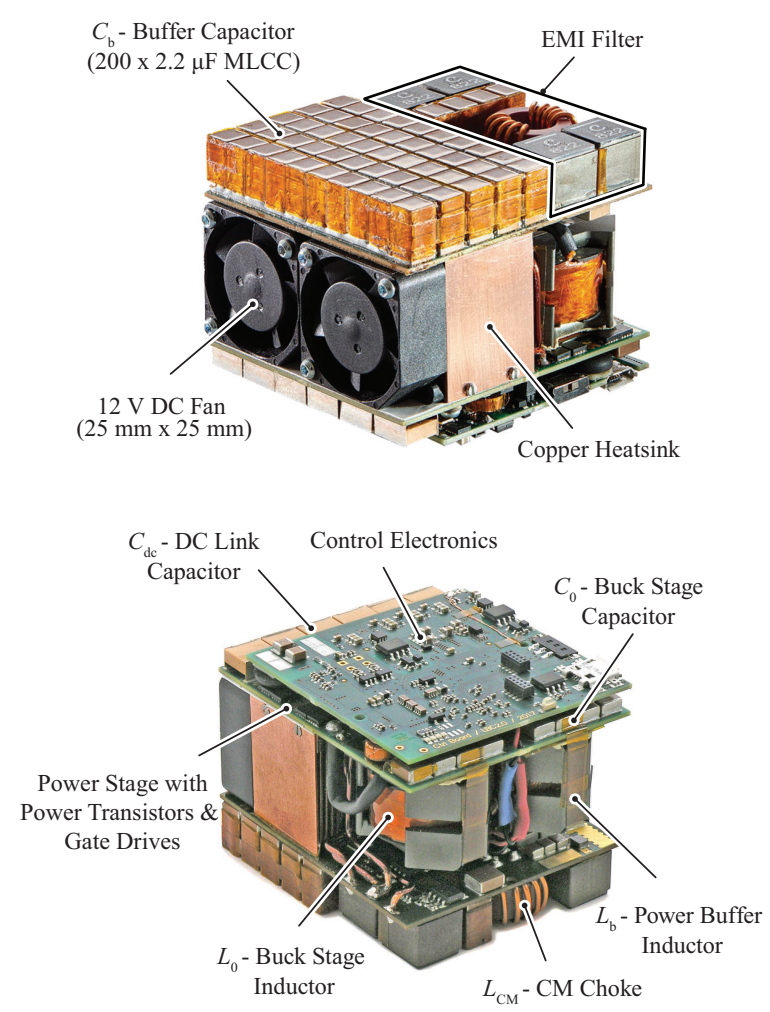

Fig. 12. Photograph of the realized Little Box 2.0 (LB 2.0) hardware (without housing) $(5.9 \mathrm{~cm} \times 5.0 \mathrm{~cm} \times 4.5 \mathrm{~cm}, 2.32 \mathrm{in} \times 1.97 \mathrm{in} \times 1.77 \mathrm{in})$.

bridge-legs, also $600 \mathrm{~V} / 70 \mathrm{~m} \Omega$ CoolGaN technology is used with two transistors in parallel per switch. In order to reduce losses during reverse conduction of the GaN transistors, $600 \mathrm{~V}$ SiC Schottky diodes are used. The inductor of the buck-stage converter, $L_{1}=45 \mu \mathrm{H}$ is implemented on a RM 10 core using the MnZn ferrite material N97 from TDK. The winding is realized with 30 turns of a $180 \times 71 \mu \mathrm{m} \mathrm{HF} \mathrm{litz} \mathrm{wire} \mathrm{without}$ additional silk insulation to achieve a higher copper filling factor. The limbs of the RM 10 core were shortened with a diamond wheel precision saw to achieve a total air gap length of $6 \mathrm{~mm}$ ( $3 \mathrm{~mm}$ per limb) while keeping the total height of the core unchanged. The inductor of the PCI, $L_{\mathrm{b}}=40 \mu \mathrm{H}$, is also implemented on a N97/RM 10 core. The winding is 


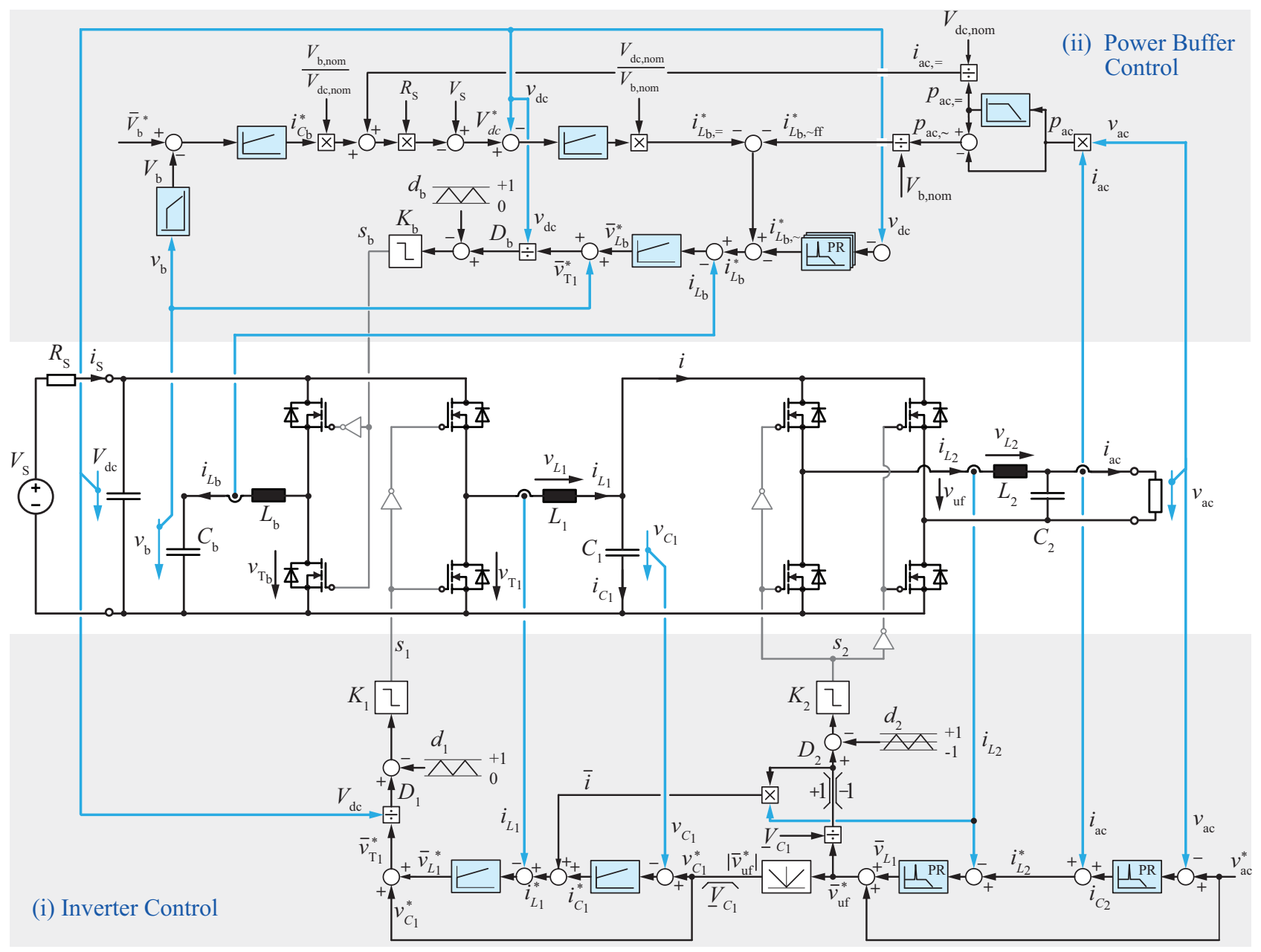

Fig. 13. Implemented digital control system of the Little Box 2.0 (LB 2.0). The inverter control structure is located below and the power buffer control structure is located above the power circuit diagram of the converter. For the sake of simplicity, the complete EMI filter is not included in the circuit diagram. The AC output voltage is controlled in the DM filter at the unfolder output, which is represented by components $L_{2}$ and $C_{2}$.

realized with 20 turns of a $225 \times 71 \mu \mathrm{m}$ HF litz wire also without additional silk insulation. The limbs of the RM 10 core were also shortened to achieve a total air gap length of 2 $\mathrm{mm}$ (1 mm per limb) while keeping the total height of the core unchanged. To minimize the conduction losses of the H-bridge unfolder, $650 \mathrm{~V} / 25 \mathrm{~m} \Omega$ enhancement-mode GaN transistors from GaNSystems (GS66516T) are employed as they feature best-in-class on-state resistance. Because the hardware design does not allow for a direct heat sink connection, two $25 \mathrm{~m} \Omega$ transistors are connected in parallel per switch to further reduce the conduction losses and virtually render cooling unnecessary. The PCI and inverter buck-stage are operated with an EMI friendly constant $140 \mathrm{kHz}$ switching frequency. The unfolder $\mathrm{H}$-bridge is operated with $120 \mathrm{~Hz}$ except, as described in more detail in the next section, during temporary $140 \mathrm{kHz}$ unipolar PWM operation around the ZCs of the AC voltage (cf. Fig. 11 of Part A).

The capacitor of the PCI $C_{\mathrm{b}}$, with an effective large-signal equivalent capacitance of $\approx 120 \mu \mathrm{F}$, was realized by means of 200 individual $2.2 \mu \mathrm{F}, 450 \mathrm{~V}$ class II/X6S MLCCs. As can be seen in Fig. 12, 200 of these chip capacitors were soldered together on a PCB which is on the one hand a very challenging assembly task and on the other hand bears the risk of electrical failures due to micro-cracks in the ceramic material caused by mechanical stress during assembly and/or operation.

\section{B. Digital Control}

The deployed control system of the LB 2.0 prototype is depicted in Fig. 13. The structure of the implemented PCI control system is identical to the LB 1.0 prototype(cf. Fig. 5) except that the current in inductor $L_{\mathrm{b}}$ is now regulated by a conventional PI-feedback loop and fixed frequency PWM instead of TCM control. Likewise to the LB 1.0 control scheme, the AC output voltage is tightly regulated with a cascade of outer-loop voltage and inner-loop current feedback control using PR-controllers as shown in the bottom right of Fig. 13. The output voltage of the buck-stage, $v_{C_{1}}$, is regulated with a PI-compensator to follow the rectified unfolder voltage reference $\left|v_{\text {uf }}^{*}\right|$ until it falls below the minimum defined value $\underline{V}_{C_{1}}$,

$$
v_{C_{1}}^{*}=\max \left(\left|v_{\mathrm{uf}}^{*}\right|, \underline{V}_{C_{1}}\right) .
$$




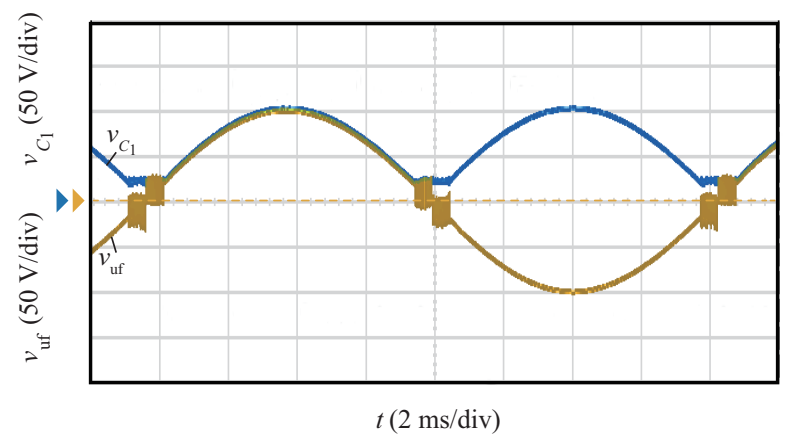

(a)

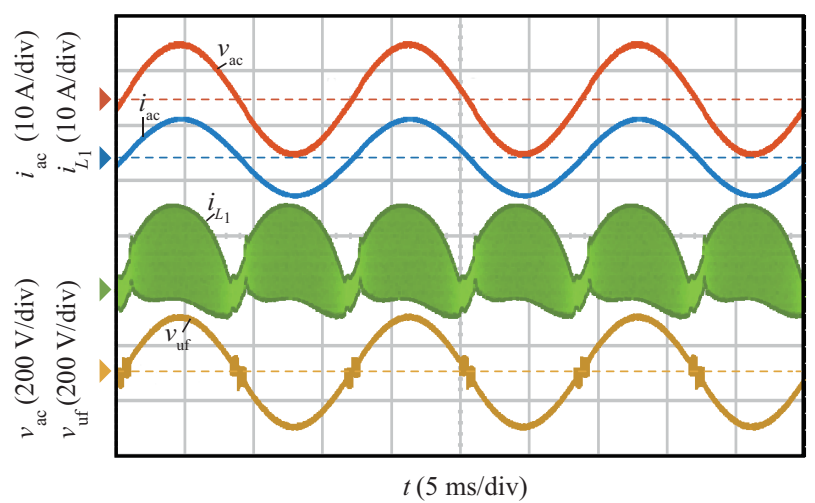

(b)

Fig. 14. Experimental measurements. (a) demonstrating the temporary PWM operation of the unfolder and the corresponding constant $\mathrm{DC} /|\mathrm{AC}|$ buck-stage output voltage $v_{C_{1}}$ which (b) prevents distortion in the AC output current during ZCs of the output voltage.

As described in Part A (cf. Fig. 11 of Part A), during the time interval when $\left|v_{\text {uf }}^{*}\right|<\underline{V}_{C_{1}}$, the buck output voltage reference is kept constant at $\underline{V}_{C_{1}}$ and the duty-cycle of the unfolder, operated with $140 \mathrm{kHz}$ unipolar PWM during this interval, is adjusted such that the voltage at the output of the unfolder $v_{\text {uf }}$ follows precisely the sinusoidal reference $v_{\mathrm{uf}}^{*}$. Preventing $v_{C_{1}}$ to fall below a minimum value, prevents distortions in the output current during the $\mathrm{ZC}$ of the voltage, which is particularly of concern for reactive loads when the output voltage and current are not in phase, and also allows to adopt TCM modulation in the buck-stage if desired $\left(v_{C_{1}}>0\right.$ needed at any time for $\mathrm{d} i_{L_{1}} / \mathrm{d} t$ $<0$ during turn-off interval). Since, the time interval in which the unfolder H-bridge is operated with PWM is very short compared to the AC period and because the switched voltage is also low $\left(=\underline{V}_{C_{1}}\right)$, the occurring switching losses of the unfolder H-bridge are negligible. The experimental waveforms to illustrate the described inverter control concept are shown in Fig. 14 and were recorded during commissioning of the LB 2.0 hardware. Fig. 14(a) shows how $v_{C_{1}}$ does not fall below the specified minimum voltage of $\underline{V}_{C_{1}}=25 \mathrm{~V}$ and the unfolder $\mathrm{H}$-bridge engages in unipolar PWM during this period. As can be seen in Fig. 14(b), this effectively prevents distortions during the $\mathrm{ZCs}$ of the $\mathrm{AC}$ output voltage typically present in $\mathrm{PFC}$ rectifier systems.

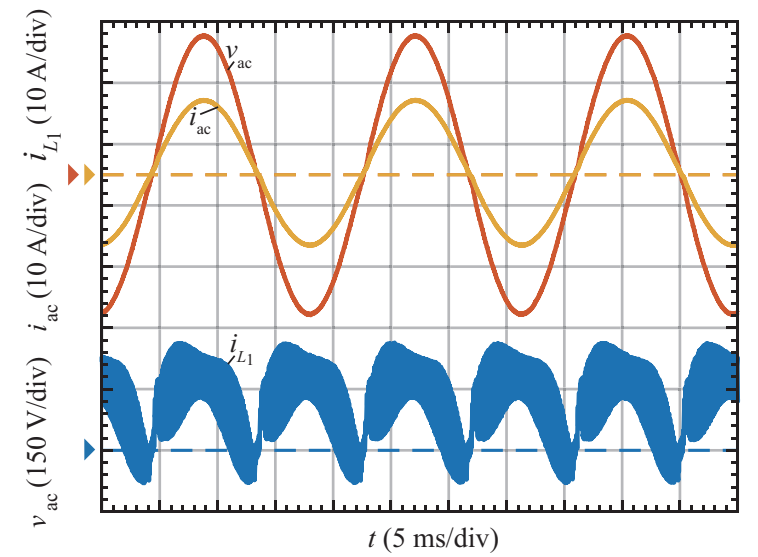

(a)

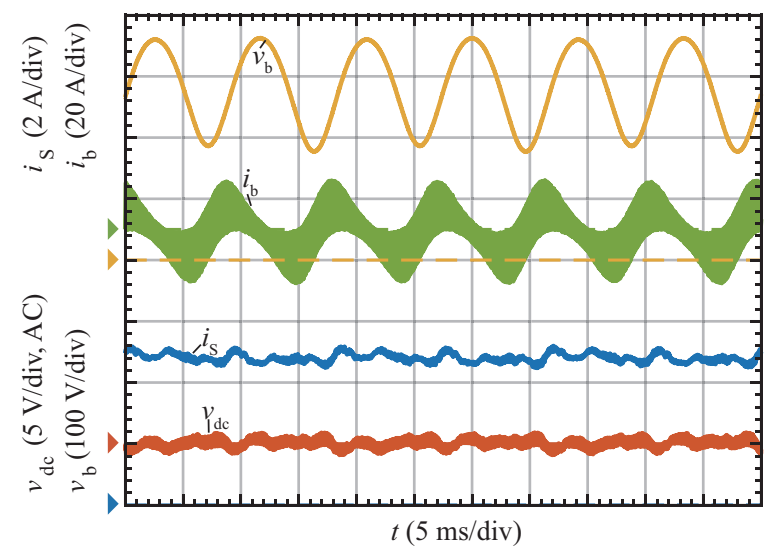

(b)

Fig. 15. Measured steady-state waveforms of the Little Box 2.0 (LB 2.0) operated at rated $2 \mathrm{~kW}$ output power $\left(\cos \varphi_{0}=1\right)$. (a) shows the AC output waveforms $v_{\mathrm{ac}}$ and $i_{\mathrm{ac}}$, and the high-frequency current in the buck-stage inductor $L_{1}$. (b) The active power buffer clearly eliminates the double-line frequency voltage ripple.

In order to tightly control $v_{C_{1}}$, particularly at the transitions between rectified sinusoidal and constant voltage reference, a cascade of PI-controllers with outer-loop voltage and innerloop current feedback is employed. Likewise to the PCI power buffer, conventional constant frequency PWM is employed to generate the switching signals for the $\mathrm{DC} /|\mathrm{AC}|$ inverter buckstage.

The entire digital control system of the LB 2.0 depicted in Fig. 13, is implemented on a TI C2000 Delfino real-time microcontroller (TMS320F28335ZJZS) and no additional FPGA is employed. The currents are measured with a Halleffect current sensor from Allegro featuring $1 \mathrm{MHz}$ bandwidth (ACS730).

\section{Experimental Waveforms}

Fig. 15 shows the measured characteristic waveforms of the LB 2.0 prototype at steady-state and rated output power. The sinusoidal output voltage and current and the HF current in the $\mathrm{DC} /|\mathrm{AC}|$ buck-stage inductor $L_{1}$ are shown in Fig. 15(a). 


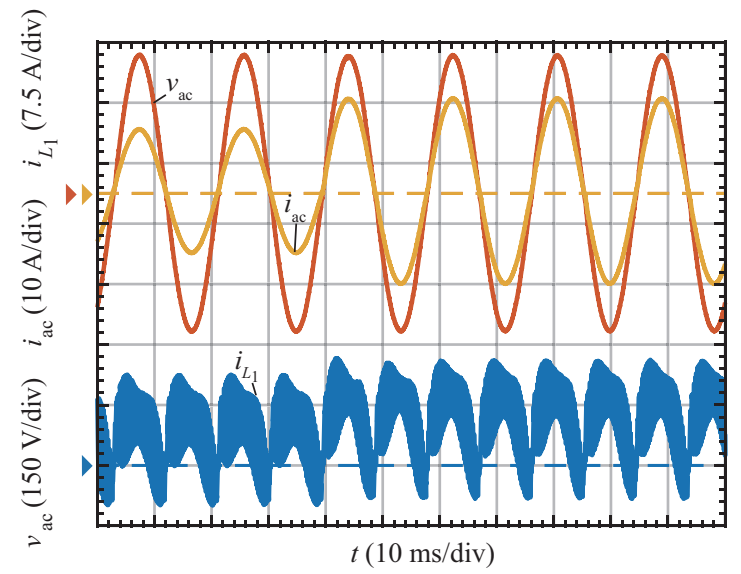

(a)

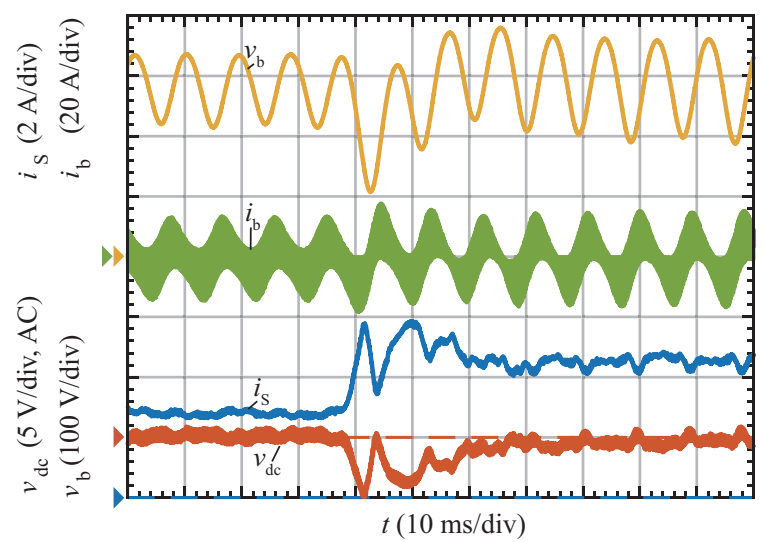

(b)

Fig. 16. Transient waveforms of the Little Box 2.0 (LB 2.0) subject to a stepwise increase in output power. (a) Measured AC output waveforms $v_{\mathrm{ac}}$ and $i_{\text {ac, }}$, and filter inductor current $i_{L_{1}}$ and (b) measured DC-side and power buffer waveforms resulting from a stepwise load increase from $1.35 \mathrm{~kW}$ to $2 \mathrm{~kW}$.

The waveforms of the PCI and the DC-side of the converter are depicted in Fig. 15(b). Considering $v_{\mathrm{dc}}$ (red trace) and converter input current $i_{\mathrm{S}}$ (blue trace), it can be seen that the PCI effectively compensates the fluctuating AC power and the specified DC-link voltage and input current ripple limits are clearly met. Note, that the measurement of $v_{\mathrm{dc}}$ was performed with AC coupling. The resulting peak-to-peak voltage swing present in the buffer capacitor voltage $v_{\mathrm{b}}$ amounts to $180 \mathrm{~V}$ and is superimposed to the bias or offset voltage $V_{\mathrm{b}}=280$ $\mathrm{V}$. Because of the lower AC excitation losses of the class II/ X6S capacitors [1], a much larger voltage swing is feasible compared to the CeraLink power buffer (cf. Section II-C, peakto-peak voltage swing of $110 \mathrm{~V}$ ). Fig. 16 shows the measured waveforms of the LB 2.0 converter during a transient caused by a stepwise increase in output power. Fig. 16(a) shows the AC output waveforms and Fig. 16(b) shows the corresponding PCI power buffer and DC-side related waveforms during a load step from $1.35 \mathrm{~kW}$ to $2 \mathrm{~kW}$. The amplitude of the AC output voltage and current are settled quickly after one period which can be attributed to the high bandwidth of the underlying $\mathrm{DC} /|\mathrm{AC}|$ buck-stage current control (cf. Section
III-B of this paper). Triggered by the step, the average buffer capacitor voltage drops around $100 \mathrm{~V}$ below the $280 \mathrm{~V}$ at steady-state and recovers after $35 \mathrm{~ms}$ when the transient is settled. Naturally, the buffer capacitor voltage exhibits a larger amplitude of the $120 \mathrm{~Hz}$ voltage ripple once the load is increased. Note that the DC-link voltage settles at the same level after the load step because of the AC coupling of the voltage measurement. As it can be inferred from the shorter settling time and the overshoot of the input current $i_{\mathrm{S}}$, the DClink controller is tuned more aggressively compared to the LB 1.0 implementation (cf. Section II-C). In any case, the transient performance of the deployed control system clearly complies with the specified settling time of $1 \mathrm{~s}$ for the maximum required step size of $500 \mathrm{~W}$.

\section{EMI Compliance}

Because of the intermittent HF PWM operation of the unfolder, a dedicated output filter for each leg of the H-bridge is provided. Since only a low voltage, $\underline{V}_{C_{1}}=25 \mathrm{~V}$, is switched with $140 \mathrm{kHz}$, a comparably small DM filter inductor $L_{\mathrm{DM} 0}$ $=8.2 \mu \mathrm{H}$ (Coilcraft XAL1010-822MED) is sufficient at the output of the unfolder. The filter capacitors placed between the output phases, $C_{\mathrm{DM} 1}$, and between the respective output phase and the negative DC-Link terminal, $C_{2}$, are implemented with $4 \times 2.2 \mu \mathrm{F} 450 \mathrm{~V} / \mathrm{X} 6 \mathrm{~S}$ MLCC. In order to dampen transients possibly triggered at the beginning and end of the recurring unfolder PWM operation interval, an additional damping branch is included in the first filter stage. The damping resistor $R_{\mathrm{DM} 1} \approx 1.25 \Omega$ is implemented with 8 parallel $10 \Omega$ 0805 SMD resistors. It should be pointed out again, that the output capacitors $C_{2}$ help to attenuate not only DM noise but also CM noise which allows to place only a single dedicated $\mathrm{CM}$ inductor in the EMI filter. $L_{\mathrm{CM} 1}$ is realized with a toroidal VITROPERM 500F core (T60006 $\neg$ L2009-W914) with 10 turns using $1 \mathrm{~mm}-\varnothing$ solid copper wire. To achieve the required $\mathrm{DM}$ attenuation, additional $\mathrm{DM}$ inductors $L_{\mathrm{DM} 1}$ are placed in the second filter stage. Likewise to $L_{\mathrm{DM} 0}$, the same commercially available $8.2 \mu \mathrm{H}$ inductors from Coilcraft are used. The $\mathrm{CM}$ capacitors $C_{\mathrm{CM} 0}$ and $C_{\mathrm{CM} 1}$ are implemented with $3 \times 100 \mathrm{nF}, 630 \mathrm{~V}$ class II/X7R MLCCs from TDK (C3225X7T2J104K160AC).

As it became evident during the experimental EMI measurements, the implemented filter as shown in Fig. 11 was not sufficient to meet the FCC Part 15 B limits at frequencies below $1 \mathrm{MHz}$. By means of measurements with a DM/CM noise separator auxiliary equipment, inserted between the LISN RF output and the test-receiver input, CM noise was identified as the main problem. To meet the EMI requirements, an additional tiny $\mathrm{CM}$ choke was inserted at the DC input of the converter as shown in Fig. 17. The additional choke $L_{\mathrm{CM} 0}$ was realized with a toroidal core with outer diameter of 13.7 $\mathrm{mm}$ and height of $3.6 \mathrm{~mm}$ using also VITROPERM 500F 


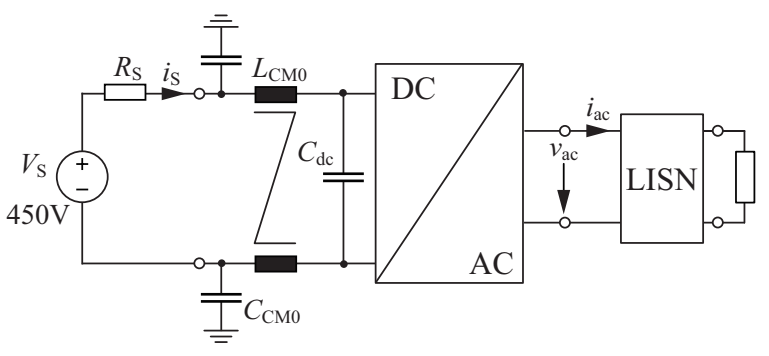

Fig. 17. Additional tiny $\mathrm{CM}$ choke $L_{\mathrm{CM} 0}$ inserted at the DC input to meet the EMI limits.

(T60006-L2012-V217). The CM winding was realized with 5 turns of $0.8 \mathrm{~mm}-\varnothing$ ) solid copper wire resulting in a nominal $\mathrm{CM}$ inductance of around $250 \mu \mathrm{H}$. The boxed volume of this additional CM choke is in the order of only $1.3 \mathrm{~cm}^{3}(<1.0 \%$ of the total LB 2.0 volume) and there is virtually no impact on the overall efficiency.

Since it was only intended to test the LB 2.0 hardware prototype in the laboratory, no explicit enclosure was designed. However, for the conducted EMI measurements presented next, the converter was placed on top of an aluminum baseplate which was connected to the ground/PE terminal of the EMI test setup. The ground terminal of the prototype was directly connected to the aluminum plate. The measured quasi-peak EMI spectrum of the LB 2.0 hardware prototype at $2 \mathrm{~kW}$ output power is depicted in Fig. 18(a). The results where again obtained with the R\&S ESPI7 test receiver and the R\&S ENV216 single-phase LISN which was installed between the $\mathrm{AC}$ output of the converter and the resistive load bank. It can be clearly seen that at low frequencies up to $20 \mathrm{MHz}$ the FCC Part 15 B conducted EMI limits are met. Compared to the spectrum of the LB 1.0 prototype (shown in light grey), distinct peaks are visible at multiples of the switching frequency. As can be seen, at very high frequencies between $20-30 \mathrm{MHz}$, the measured noise just remains below the limit. Since parasitics are detrimental to the available filter attenuation at these high frequencies, it is expected that with a complete metal enclosure several $\mathrm{dB} \mu \mathrm{V}$ of safety margin could be gained.

As described previously in Section II-D of this paper, for the ground current measurement an additional external decoupling CM choke was inserted between the DC supply (Sorensen SGI $600 / 17$ ) and the input of the converter. The nominal inductance value of this decoupling CM choke is almost a factor of 9 larger compared to $L_{\mathrm{CM} 0}$. The measured ground current at the nominal operating point is depicted in Fig. 18(b) and its RMS value amounts to $5.7 \mathrm{~mA}$ which would exceed the initial strict ground current limit of $5 \mathrm{~mA}$. Clearly visible are the current peaks up to $100 \mathrm{~mA}$ located around the zero crossings of the $\mathrm{AC}$ voltage which are triggered by imperfections in the transition to temporary PWM operation of the H-bridge unfolder and deviations of the $\mathrm{DC} /|\mathrm{AC}|$ buck-stage output voltage $v_{C_{1}}$ from its reference. Without adding the additional (external) $\mathrm{CM}$ choke between the DC supply and the input of

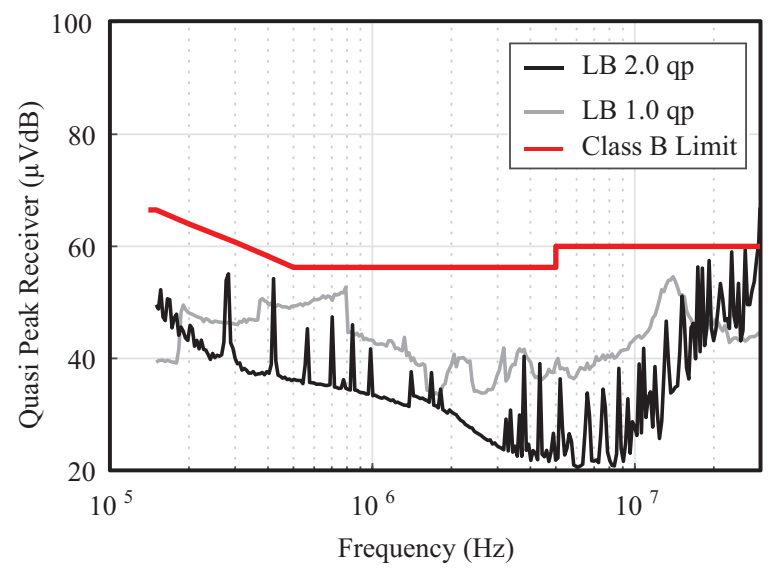

(a)

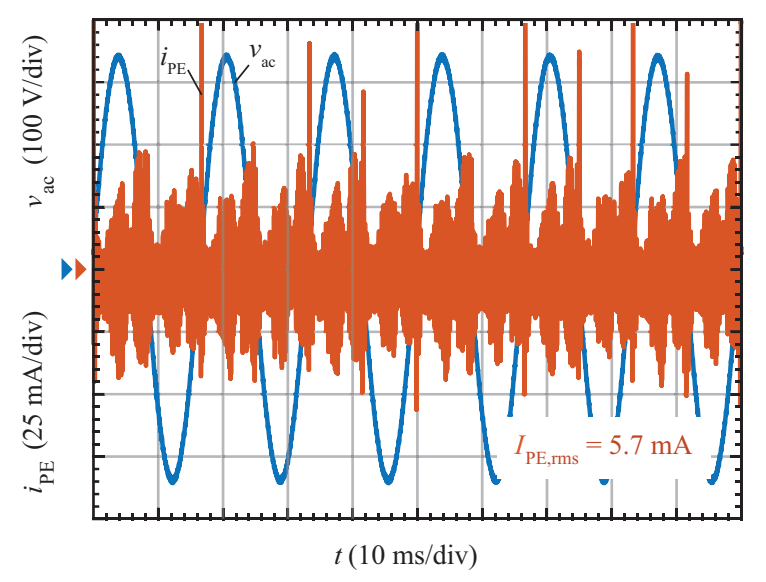

(b)

Fig. 18. (a) EMI noise spectrum of the Little Box 2.0 at $2 \mathrm{~kW}$ output power measured with R\&S ENV216 single-phase LISN and R\&S ESPI7 test receiver. (b) Ground current of the Little Box 2.0 measured by means of inserting both inverter output conductors simultaneously into the clamp of the current probe (Teledyne CP030).

the converter, the RMS value of the measured ground current amounts to $14.93 \mathrm{~mA}$ which is still in accordance with the revised GLBC specifications.

\section{E. Efficiency}

The measured efficiency of the LB 2.0 prototype as a function of output power is depicted in Fig. 19(a). The blue line with circle-type markers represents the efficiency of the system if two Sunon 12 V DC fans (MF25101V1-1000U-A99) are employed. The peak efficiency at $2 \mathrm{~kW}$ amounts to $97.4 \%$. The red line with cross-type markers represents the measured efficiency if two $8 \mathrm{~V}$ high-speed fans are employed (WTF, 20 000 RPM@7.2 V). The total power consumption of the WTF fans amounts to $\approx 6 \mathrm{~W}$ which explains the drop in efficiency to $97.2 \%$ at full output power. As indicated, this is still significantly higher than the $96.3 \%$ peak efficiency achieved with the LB 1.0 converter. The CEC weighted efficiency of the LB 2.0 amounts to $96.12 \%$ with conventional fans (Sunon). Since the increased cooling performance achieved with the 


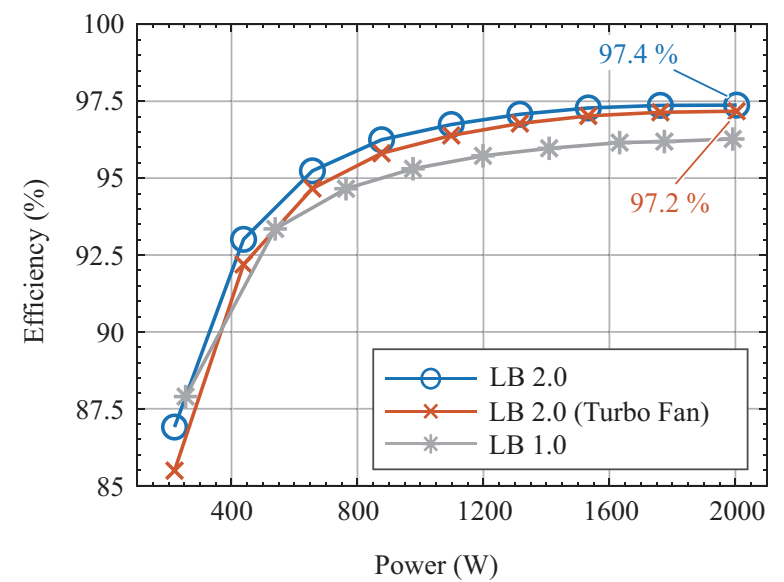

(a)

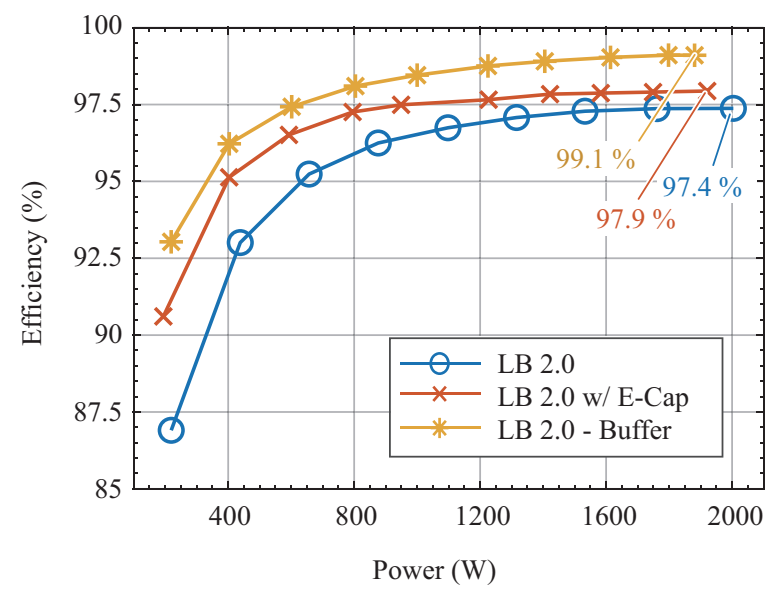

(b)

Fig. 19. Conversion efficiency of the Little Box 2.0 (LB 2.0) with respect to output power measured with a Yokogawa WT3000 precision power analyzer. (a) Efficiency with normal and high-speed DC fan and comparison with results from Little Box 1.0. (b) Efficiency with respect to output power of only the active power buffer (tested with electronic load), only the inverter equipped with electrolytic capacitors and the complete system.

high-speed fans is actually only needed at high loads above $\approx 1.7$ $\mathrm{kW}$ and the CEC weighting coefficient at rated power is only 0.05 , the lower nominal efficiency when using the high-speed fans has virtually no affect on the CEC weighted efficiency. In practice, the fan speed can be adjusted according to the output load level and/or the corresponding cooling demand (this was not considered during the experimental testing), thus it is reasonable to report a weighted efficiency of $96 \%$. The yellow line with star-type marker depicted in Fig. 19(b) represents the efficiency of only the PCI without the inverter. For this purpose, an electronic load (Chroma 63201) was used to emulate the pulsating power caused by the inverter. It can be seen that the peak efficiency at close to rated output power exceeds $99 \%$ efficiency. Displayed in red with crosstype markers is the measured efficiency of the LB 2.0 if only the inverter is operated and the DC-link is passively buffered with high-density electrolytic capacitors $(3 \times 493 \mu \mathrm{F}, 450 \mathrm{~V}$, B43991-X0009-A223 from EPCOS/TDK). As can be seen in the plot, the peak efficiency then almost reaches $98 \%$ at full output power. Due to the high efficiency of the PCI close to rated power, replacing the electrolytic capacitors with the active power buffer only reduces the overall efficiency by roughly $0.5 \%$ In case of the LB 1.0 (cf. Section II-E), substituting the electrolytic capacitors with the more compact PCI resulted in a reduction of $1.2 \%$ in overall efficiency. The main reason for this is that compared to the CeraLink technology utilized in the LB 1.0 to realize the buffer capacitor, the X6S MLCC exhibits a much lower power loss $(\approx 1.5 \mathrm{~W}$ instead of $\approx 17.3 \mathrm{~W}$ at $2 \mathrm{~kW})$.

\section{F. Cooling and Operating Temperature}

As can be seen from the picture of the LB 2.0 hardware in Fig. 12, the cooling system consists of a single custom machined copper heat sink and two $25 \mathrm{~mm} \times 25 \mathrm{~mm} \times 10 \mathrm{~mm}$ DC fans. The fans are directly attached to the fins of the heat sink without an additional duct. The $4 \mathrm{~mm}$ thick base-plate extracts the losses from the power transistors (cooling through the PCB). The air stream exiting the heat sink, is then cooling the power inductors $L_{1}$ and $L_{\mathrm{b}}$ which are located directly after the heat sink. The fin dimensions are $20 \mathrm{~mm} \times 18 \mathrm{~mm} \times 0.5$ $\mathrm{mm}$ and the channel width (distance between fins) amounts to $0.8 \mathrm{~mm}$. Since manufacturing from a single copper block was not possible due to mechanical limitations, all fins were first cut from a thin $0.5 \mathrm{~mm}$ copper sheet and then inserted into machined channels in the baseplate and afterwards soldered for permanent fixation. Considering Sunon $12 \mathrm{~V}$ DC fans (MF25101V1-1000U-A99), the calculated cooling system performance index (CSPI, cf. Section III-D of Part A) is in the order of $53 \mathrm{~W} /\left(\mathrm{K} \mathrm{dm}^{3}\right)$. Treating the free space around the power inductors as air duct (cf. Fig. 12), the performance reduces to $37.5 \mathrm{~W} /\left(\mathrm{K} \mathrm{dm}^{3}\right)$.

In order to further increase the airflow passing by the power inductors and/or to limit the maximal winding temperature of the power inductors, the Sunon fans were replaced with more powerful ultra-high speed fans from WTF (20 000 RPM, repurposed from RC model car applications) to provide better cooling in the nominal operating point as discussed previously.

The thermography image in Fig. 20 was recorded with a FLIR A655sc HD infrared camera and shows the steady-state temperature distribution of the LB 2.0 at $2 \mathrm{~kW}$ output power from two perspectives. The average temperature of the power stage (power transistor, gate drives, etc.) is approximately $80{ }^{\circ} \mathrm{C}$. The power inductor of the inverter generates more losses and therefore, exhibits a higher steady-state operating temperature. The temperature of the winding reaches approximately $84{ }^{\circ} \mathrm{C}$. However, it should be noted that surfaces exposed to the outside which would directly touch the metallic enclosure, such as the core of the power inductors, the buffer capacitor, the EMI filter, the heat or the control PCB, exhibit operating temperatures of only $50-65^{\circ} \mathrm{C}$.

\section{Discussion \& Performance Benchmark}

Based on the experimental results for the two hardware demonstrators provided in the previous sections, it can be 


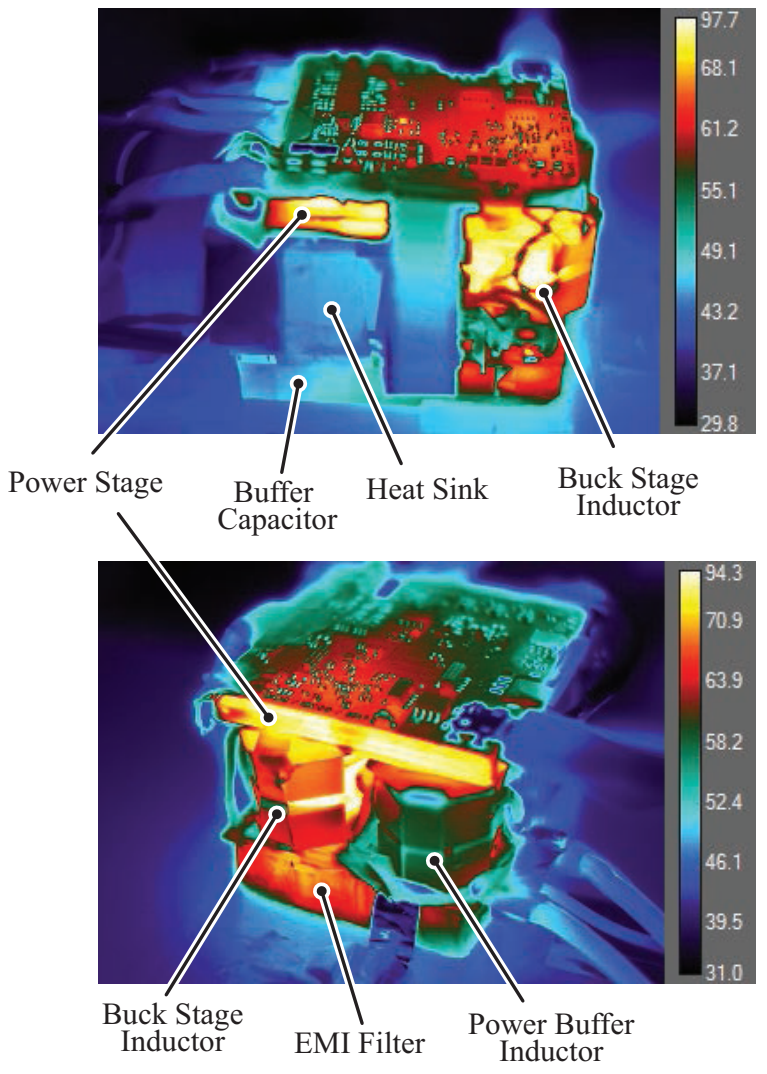

Fig. 20. Thermography images of the Little Box 2.0 (without enclosure) recorded with a FLIR A655sc infrared camera showing the steady-state temperature distribution at rated $2 \mathrm{~kW}$ output power.

concluded that both systems meet the technical specifications of the GLBC. Fig. 21 shows the calculated loss and measured volume distribution at $2 \mathrm{~kW}$ output power of the realized prototypes. As the total volume and loss figures indicate, 18 $\mathrm{W}$ of losses and about $103 \mathrm{~cm}^{3}$ of volume are saved in case of the improved LB 2.0 compared to the LB 1.0. Because of the reduced power loss of the components and the higher cooling system performance, the cooling volume of the LB 2.0 was reduced from $95 \mathrm{~cm}^{3}(39.6 \%)$ to $46.5 \mathrm{~cm}^{3}(33.7 \%)$. Due to the installed ultra-high speed fans for improved cooling of the LB 2.0, the power consumption of the auxiliary electronics has almost doubled.

\section{A. Main Reasons for the Higher Performance of the LB 2.0}

As can be noticed by comparing Fig. 21(a) and (b), the power loss of the buffer capacitor (shown in dark blue) was significantly reduced. As described in Section II of this paper, the buffer capacitor of the LB 1.0 was implemented with 120 x $2 \mu \mathrm{F}, 600 \mathrm{~V}$ CeraLink capacitors from EPCOS/TDK. These ceramic capacitors can be operated with a large current ripple and feature a high capacitance density which, in contrast to class II ceramics, even increases with applied bias voltage and/ or temperature [11]. Furthermore, the capacitors are available in compact $20 \mu \mathrm{F}$ or even custom-made packages making the system assembly much easier and more reliable. From this perspective, the CeraLink technology seemed to be the right choice to implement the PCI buffer in the course of the GLBC, however, it turned out that these capacitors unfortunately generate high losses when subjected to a LF AC voltage with large amplitude such as present across the PCI buffer capacitor. For this reason, in the PCI buffer of the LB 2.0 system, class II/ X6S MLCCs were utilized resulting in a reduction of the buffer capacitor losses by $15.8 \mathrm{~W}$. Because of the lower loss density it is also possible to operate the buffer capacitor with a larger LF AC voltage amplitude of the voltage ripple which results in a slightly lower volume despite the fact that the capacitance density of the X6S MLCCs is slightly lower compared to CeraLink capacitors.

The LB 1.0 H-bridge inverter with 4D-interleaving was implemented with a total of 4 bridge-legs each with a dedicated HF inductor. In contrast, the LB 2.0 inverter DC/|AC|buckstage was realized with a single bridge-leg and a single HF inductor. Therefore, despite the much lower switching frequency of the LB $2.0,140 \mathrm{kHz}$ as opposed to $250 \mathrm{kHz}^{-1}$ $\mathrm{MHz}$ of the LB 1.0, the total volume of the inverter's power stage - mainly determined by the power transistors and filter inductors - was reduced by roughly $50 \%$ from $40 \mathrm{~cm}^{3}$ to 19.3 $\mathrm{cm}^{3}$. Interestingly, despite ZVS throughout the AC period and 4D-interleaving in case of the LB 1.0, the total losses in the power transistor are considerable due to the much higher switching frequency. As analyzed in [24] and discussed in Part A of this publication, for the employed $600 \mathrm{~V} / 70 \mathrm{~m} \Omega \mathrm{GaN}$ Gate Injection Transistor (GIT), soft-switching of $10 \mathrm{~A}$ at 400 $\mathrm{V}$ causes $2 \mu \mathrm{J}$ of energy dissipation per transistor and switching cycle. To exemplify, for a switching frequency of $1 \mathrm{MHz}$, this translates into $4 \mathrm{~W}$ of losses per bridge-leg and thus ZVS losses cannot be neglected at very high switching frequencies even if the latest GaN semiconductor technology is employed. It has been identified [24], that the lossy charging and discharging of the transistors' output capacitance $\left(C_{\text {oss }}\right)$ during the resonant transition is the origin of the experienced ZVS losses and that the dissipated energy is a function of applied $\mathrm{d} v_{\mathrm{ds}} / \mathrm{d} t$ rather than switched current. For this reason, practically lossless class I/ C0G MLCC capacitors $C_{\text {ext }}=600 \mathrm{pF}$, were added in parallel to the high-side and low-side GITs (charge equivalent parasitic output capacitance $C_{\text {oss, Qeq }}=114 \mathrm{pF}$ ), which allowed to reduce the turn-off losses by around 30\% [3]. However, due to the higher effective output capacitance $C_{\text {oss,eff }}$, now for the ZVS transient a higher amount of charge $Q_{\text {oss,eff }}$ is needed to charge/ discharge the effective output capacitances $C_{\text {oss,eff }}$. This means that for a defined maximum dead time interval of $T_{\mathrm{dt}} \approx 125$ ns (for two transitions this corresponds to $25 \%$ of a $1 \mathrm{MHz}$ switching cycle), a large current of $5 \mathrm{~A}$ is required to charge $C_{\text {oss,eff }}$ from $0 \mathrm{~V}$ to $400 \mathrm{~V}$ and vice versa. This increases the peak-to-peak amplitude of the TCM current and therefore the RMS value and the associated conduction losses. Moreover, because of the remaining ZVS losses, a permanently interleaved operation of the bridge-legs at low power turned out to be no more beneficial in terms of efficiency. For this reason, the 4D-interleaving scheme as introduced in Part A with simultaneous operation of both bridge-legs only around the peak of the instantaneous output power was adopted. 
Cooling Housing Others Power Transistor EMI Filter Electronics Inductor Buffer Capacitor

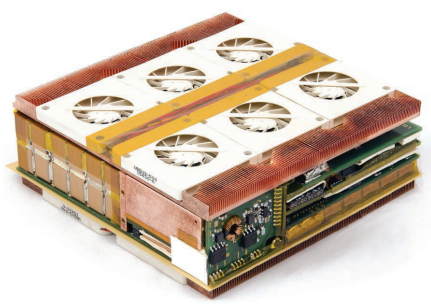

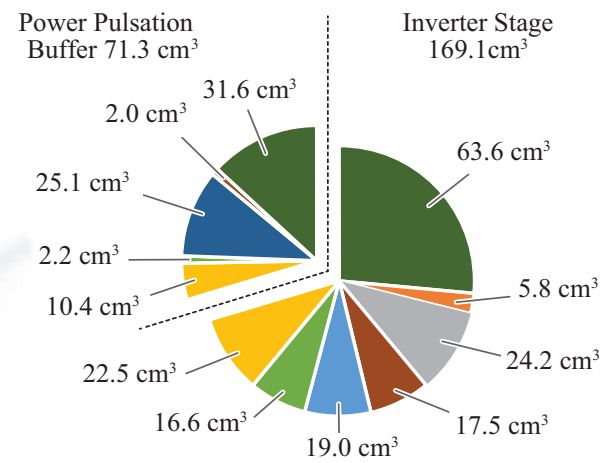

Total Volume: $240.5 \mathrm{~cm}^{3}$

(a)

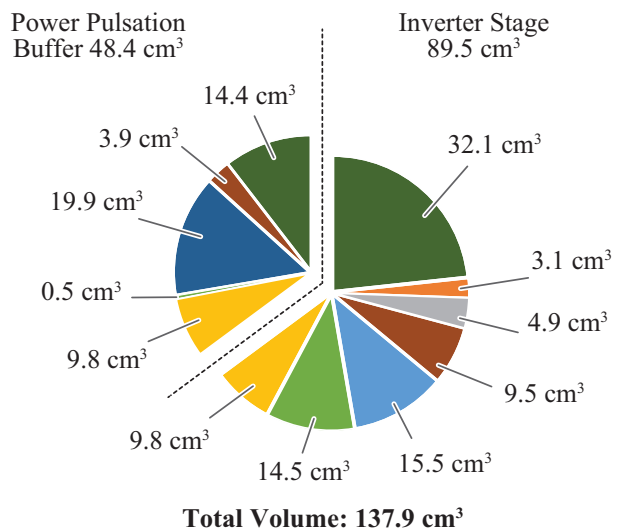

(b)

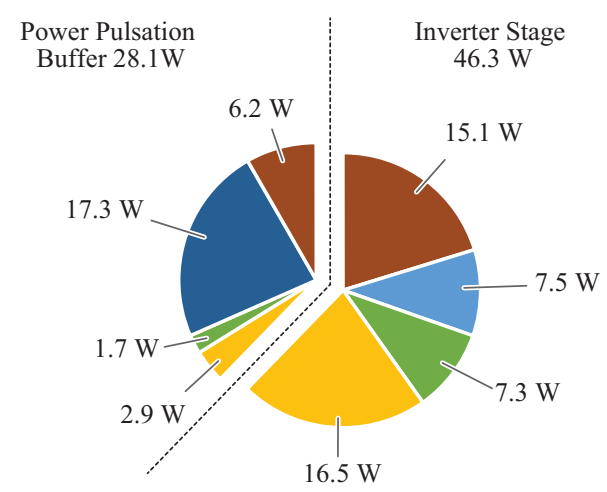

Total Losses: $74.4 \mathrm{~W}$

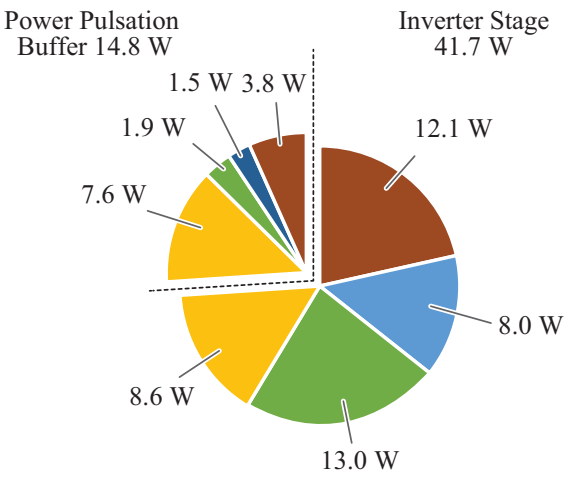

Total Losses: 56.5W

Fig. 21. Volume and power loss balance at $2 \mathrm{~kW}$ output power of the realized hardware prototypes. (a) Little Box 1.0 (LB 1.0). (b) Little Box 2.0 (LB 2.0).

In contrast, the inverter of the LB 2.0 with just a single bridge-leg and conventional PWM exhibits similar power transistor losses. This can be explained by the lower RMS value of the inductor current as opposed to the TCM modulation which results in lower conduction losses and the moderate $140 \mathrm{kHz}$ switching frequency also limits the occurring hardswitching losses.

\section{B. Benchmark With Google Little Box Challenge Finalists}

Out of $100+$ teams worldwide which submitted detailed descriptions of their technical approach, 18 teams were selected to submit their converter to Google for final testing at the National Renewable Energy Laboratory (NREL) in Golden, Colorado back in October 2015. The 1st price of the GLBC was awarded in February 2016 to the team of the Belgium company CE+T Power. Their converter achieved a power density of $8.72 \mathrm{~kW} / \mathrm{dm}^{3}\left(142.9 \mathrm{~W} / \mathrm{in}^{3}\right)$ which is -not surprisingly - only slightly higher than the power density of the LB 1.0 converter system presented in Section II of this paper, $8.18 \mathrm{~kW} / \mathrm{dm}^{3}\left(134 \mathrm{~W} / \mathrm{in}^{3}\right)$, since both teams were following the same technical approach (interleaved, H-bridge TCM inverter and buck-type PCI buffer). Among the 18 finalists, there were three categories: 7 teams were affiliated with technical consulting firms, 7 teams were affiliated with electrical engineering companies and only 4 teams were affiliated with universities. Moreover, 3 out of the 18 finalists did not show up at the final event. Most of the teams used either a H-bridge or a $\mathrm{DC} / \mathrm{AC} \mid$ buck-stage and $\mid \mathrm{AC} / \mathrm{AC} \mathrm{H}$-bridge unfolder topology to implement the inverter but overall no fundamentally new approach was presented. One of several innovative approaches worth mentioning, is the topology presented by the University of Illinois at Urbana-Champaign (UIUC), where the $\mathrm{DC} /|\mathrm{AC}|$ buck-stage was realized with a 7-level flying capacitor topology using $100 \mathrm{~V}$ GaN transistors [25], [26]. Overall, 11 teams employed GaN power transistors, 2 teams used $\mathrm{SiC}$ and 2 teams relied on Si power transistors. Both, TCM modulation with ZVS throughout the AC period and variable switching frequency as well as conventional PWM with ZVS only around the $\mathrm{ZC}$ of the $\mathrm{AC}$ current and constant switching frequency were adopted by the finalists. Moreover, the majority of finalists used an active power buffer to cope with the fluctuating power at the AC side. The buck-type PCI buffer presented herein was employed by many finalists but also the partial-power stacked and series-connected active power buffer concepts were adopted [27], [28]. The majority of contestants 


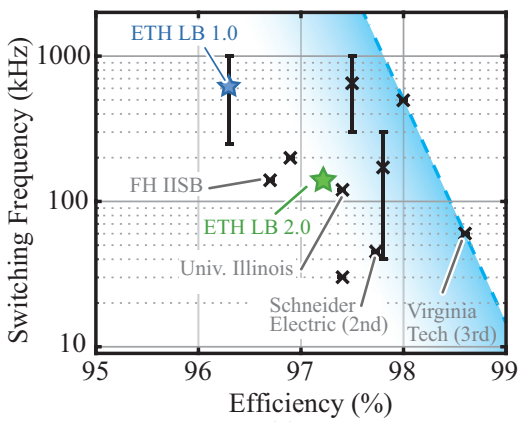

(a)

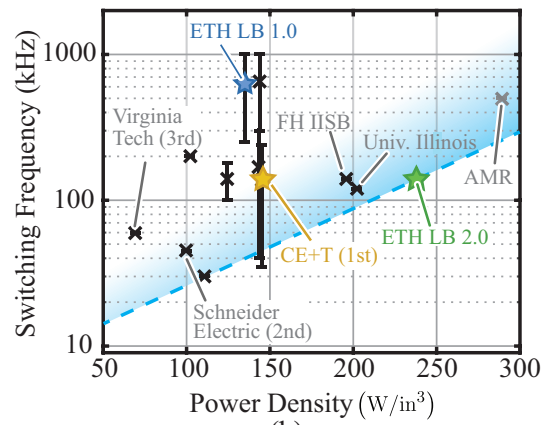

(b)

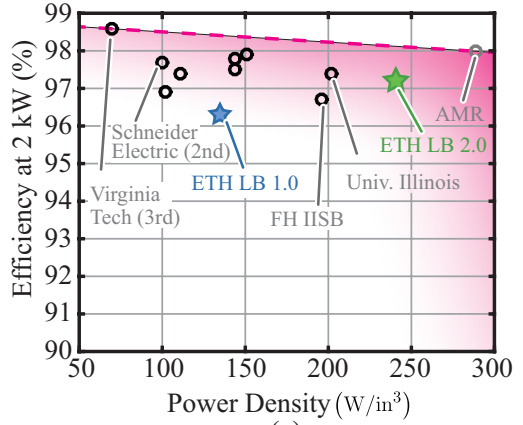

(c)

Fig. 22. Performance benchmark of the Google Little Box Challenge (GLBC) finalists concepts. The data was gathered from the technical approach documents of the finalists published by Google. Claimed performance (a) Switching frequency with respect to efficiency at $2 \mathrm{~kW}$. (b) Switching frequency with respect to power density. (c) Efficiency at $2 \mathrm{~kW}$ with respect to power density. Note that the nominal efficiency at $2 \mathrm{~kW}$ of the inverter from CE+T Power is not known.

selected the $2.2 \mu \mathrm{F}, 450 \mathrm{~V}$, class II/X6S MLCC technology for the implementation of the buffer capacitor, in the bridgeleg output filters and for the DM capacitors in the EMI filter. To implement the HF filter inductors, a conventional approach using HF litz wire and MnZn ferrite core material was adopted by most of the finalists. Only two teams, including the authors employed a multi-gap inductor design.

Likely due to the short competition time-frame of just 15 months, none of the presented prototypes relied on advanced 3D-integration techniques such as PCB-embedding of active and/or passive components [29]. Instead, the presented hardware prototypes were altogether hand crafted engineering jewels with stacked, multi-board SMD-technology-based PCB designs with careful 3D CAD mechanical arrangement of components and a sophisticated heat management. More technical details of the individual concepts of the finalists are presented in the survey in [30].

The data for the benchmark shown in Fig. 22 was gathered from the publicly available technical approach documents of the GLBC finalists. Note, that some teams did not publish the achieved peak efficiency values are therefore not included in Figs. 22(a) and (c). Figs. 22(a) and (b) show the switching frequency with respect to the efficiency at $2 \mathrm{~kW}$ and with respect to the achieved power density, respectively. The interval marker indicates the variable switching frequency resulting from TCM modulation. Several teams selected a design with comparably high switching frequency in the range of 250 $\mathrm{kHz}^{-1} \mathrm{MHz}$, others with a much lower switching frequency in the range of 35-300 kHz. Irrespective of the frequency range, as can be seen in Fig. 22(b), the systems employing TCM reached similar power densities around $8.54 \mathrm{~kW} / \mathrm{dm}^{3}(140$ $\mathrm{W} / \mathrm{in}^{3}$ ). Interestingly, two teams, i.e. Fraunhofer Institute for Integrated Systems and Device Technology (FH IISB) and the UIUC, achieved a remarkable power density around $200 \mathrm{~W} / \mathrm{in}^{3}$ with a comparably low switching frequency between 120-140 $\mathrm{kHz}$. In case of the 7-level flying-capacitor converter, $120 \mathrm{kHz}$ represents the switching frequency of a single converter cell and the effective switching frequency is actually $720 \mathrm{kHz}$. The data in Figs. 22(a) and (b) agrees with the well known tendency that increasing the switching frequency results in higher power density at the cost of a lower efficiency. The achieved effi- ciency with respect to power density is depicted in Fig. 22(c) and shows a clear trade-off between efficiency $\eta$ and power density $\rho$. The majority of the teams achieved a nominal efficiency in the range of $97 \%-98 \%$. Indicated with a blue and green star-type marker is the performance of the LB 1.0, described in Section II, and the improved LB 2.0, described in Section III, respectively. The yellow star marker indicates the $\eta \rho$-performance of the GLBC winners (CE+T Power). It can be seen that by means of the $\mathrm{DC} /|\mathrm{AC}|$ buck-stage based inverter topology and several improvements discussed previously, the LB 2.0 converter clearly outperforms the winning team from $\mathrm{CE}+\mathrm{T}$ Power in terms of power density. Interestingly, several teams including AMR (cf. Fig. 22(c)), OKE-Services and Cambridge Active Magnetics [30], claimed even higher power densities in the range of $300 \mathrm{~W} / \mathrm{in}^{3}$, however, no rigorous experimental evidence was published to support these claims.

\section{A Critique of Little Box Challenge Inverter Designs-Revisited}

In [31] a well written and comprehensive analysis of the GLBC inverter designs is provided. However, especially considering the latest progress achieved with the LB 2.0 hardware prototype presented herein, certain statements are questioned in the following. Fig. 23 shows the CEC weighted efficiency with respect to power density of the GLBC participants. The empirical trend-line of the observed trade-off between efficiency and power density of the GLBC inverter designs proposed in [31],

$$
\eta=100-(0.04 \times \rho),
$$

whereby $\rho$ is the power density in $\mathrm{W} / \mathrm{in}^{3}$ and $\eta$ is in $\%$, is indicated by the dashed red line in Fig. 23. Although the proposed $\eta-\rho$ trend-line is valid for many of the implemented designs, it fails to characterize the inverter designs with maximal power density and does not distinguish between the H-bridge based and $\mathrm{DC} /|\mathrm{AC}|$ buck-stage based inverter topologies. Therefore, based on the insights gained from the conducted Pareto optimization (cf. Section IV-A of Part A), two additional $\eta-\rho$ trend-lines (11) and (12) are proposed, which better describe the inverter designs of the GLBC finalists with highest power 


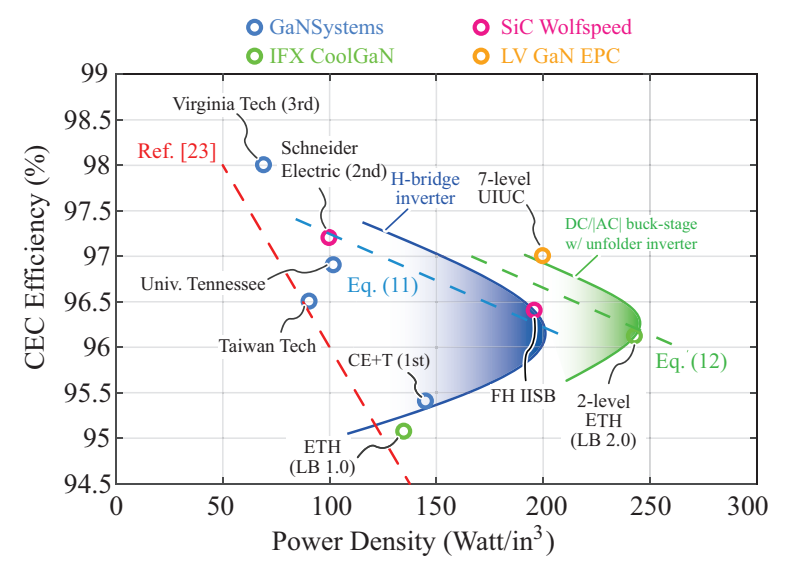

Fig. 23. CEC efficiency with respect to power density of selected Google Little Box Challenge (GLBC) participants. The efficiency and power density trade-off trend-line proposed in [31] is indicated by the dashed red line. Based on the insights gained from the Pareto optimization as presented in Part A of the paper, two additional hypothetical trend-lines for the H-bridge (dashed blue line) and the $\mathrm{DC} /|\mathrm{AC}|$ buck-stage (dashed green line) based inverter concepts, more characteristic for the designs of the GLBC finalists with highest power density are proposed. Note, that only the inverter of the GLBC participants with known CEC efficiency figures are shown.

density, i.e. FH IISB for the H-bridge based inverter and UIUC for the $\mathrm{DC} /|\mathrm{AC}|$ buck-stage based inverter concept, as indicated with the dashed blue and green lines in Fig. 23, respectively.

$$
\begin{gathered}
\eta=98.3-(0.0105 \times \rho), \\
\eta=98.75-(0.0105 \times \rho),
\end{gathered}
$$

It should be noted that this trend-lines are just hypothetical and have yet to be confirmed.

It is therefore important to underline, that the 7-level flying capacitor inverter design should not be considered as an outlier with respect to the H-bridge based designs as stated in [31] but rather as a design which follows the $\eta-\rho$ trade-off characteristic of the $\mathrm{DC} /|\mathrm{AC}|$ buck-stage based inverter topologies.

Furthermore, the statement in [31] that employing Infineon CoolGaN devices in the inverter results, in low efficiency and just slightly higher power density compared to other GaN-based H-bridge designs is clearly challenged. It can be seen from Fig. 23, that in case of the LB 2.0 inverter which employs the same CoolGaN devices as the LB 1.0 prototype, both $\eta$ and $\rho$ was increased. In this respect, it is important to emphasize that a holistic consideration of the employed topology and of all power electronic constituents is necessary to get a complete picture and thus understand the main driver of system performance. The consideration of only the power semiconductors is not sufficient. This is also exemplified by the $\eta \rho$-Pareto front simulation study depicted in Fig. 24 carried out for the H-bridge TCM inverter. In this study, scaling factors $k_{\mathrm{c}} \in[0,1]$ and $k_{\mathrm{s}} \in[0,1]$ of the transistor conduction and switching losses, respectively, are introduced to gradually idealize the power semiconductor properties. $\left(k_{\mathrm{c}}=1, k_{\mathrm{s}}=1\right)$ represents the real $\mathrm{GaN}$ transistors (Infineon CoolGaN) and $\left(k_{\mathrm{c}}=0, k_{\mathrm{s}}=0\right)$ represents idealized switches without any

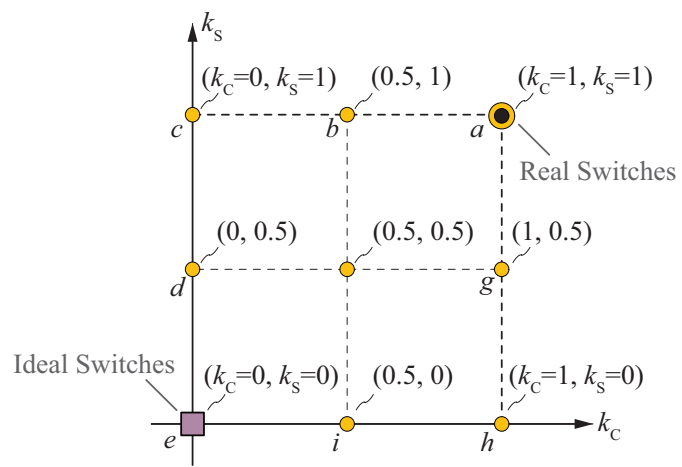

(a)

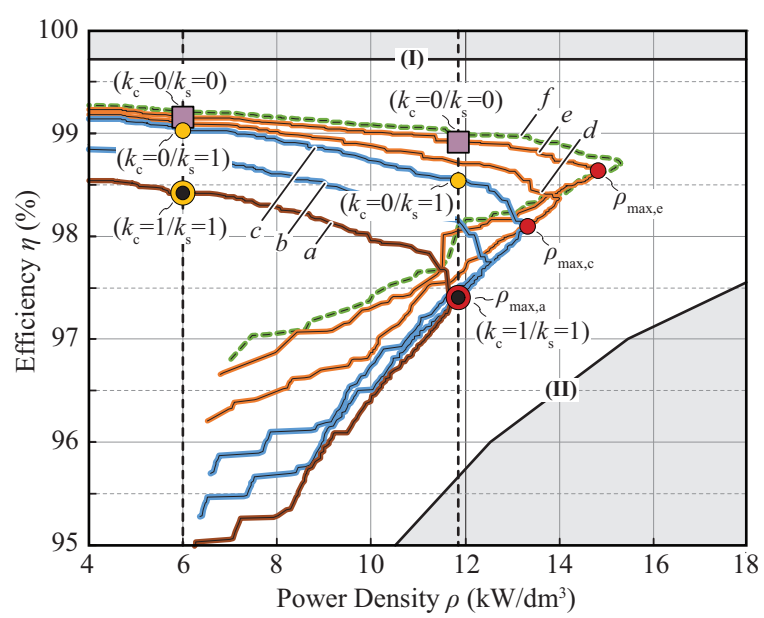

(b)

Fig. 24. (a) Scaling factors $k_{\mathrm{c}} \in[0,1]$ and $k_{\mathrm{s}} \in[0,1]$ of the conduction and switching losses, respectively, used to gradually idealize the power transistor properties; $\left(k_{\mathrm{c}}=1, k_{\mathrm{s}}=1\right)$ represents the real GaN transistors (Infineon CoolGaN), $\left(k_{\mathrm{c}}=0, k_{\mathrm{s}}=0\right)$ represents idealized switches without any conduction and switching losses. (b) The $\eta \rho$-Pareto fronts associated with the inscribed coordinates $\left(k_{\mathrm{c}}, k_{\mathrm{s}}\right)$ for the H-bridge inverter with Triangular Current Mode (TCM) operation (cf. Fig. 3 of this paper).

conduction and switching losses. It can be seen in Fig. 24(b) how idealizing the GaN transistors gradually improves both $\eta$ and $\rho$. Once conduction, switching and gate drive losses of the power semiconductors are completely excluded (ideal switches), the $\eta \rho$-Pareto front is exclusively determined by the filter passives, heat sink and auxiliary electronics. Naturally, if these components are implemented poorly, the resulting $\eta \rho$ performance is moderate even if ideal semiconductors would be at one's disposal.

\section{Concerning an Industrial Implementation}

The components of the realized hardware prototypes presented in this paper are all operated within their safe operating area recommended by the manufacturers. The electrical distances in the PCB layouts satisfy laboratory requirements but do not comply with industrial creepage distance requirements. However, this can be addressed with a higher level of integration and advanced packaging. Although the employed high energy density ceramic capacitors significantly improve power density, they are by no means an option for a cost- 
sensitive application. To exemplify, the active power buffer of the LB 2.0 uses 200 pieces of the $2.2 \mu \mathrm{F}$ class II/X6S MLCC which amounts to $\$ 290$ of component cost for the buffer capacitor (Digikey, order quantities above 1000 pieces). In contrast, using electrolytic capacitors for passive buffering of the DC-link amounts to only $\$ 14.85$ of component cost $(3 \times$ $470 \mu \mathrm{F}$ from TDK's ultra-compact $450 \mu \mathrm{F}$ B43640 series, at least $1.1 \mathrm{mF}$ needed to satisfy the voltage ripple requirement). This cost consideration is likely also the reason behind Schneider Electrics' series-connected partial-power buffering approach which only relies on electrolytic capacitors [28]. In case of the LB 2.0, if electrolytic capacitors are employed instead of the active power buffer, then the power density would drop from $14.8 \mathrm{~kW} / \mathrm{dm}^{3}\left(243 \mathrm{~W} / \mathrm{in}^{3}\right)$ to approximately $9.48 \mathrm{~kW} / \mathrm{dm}^{3}\left(155.4 \mathrm{~W} / \mathrm{in}^{3}\right)$ considering a boxed volume of $121.5 \mathrm{~cm}^{3}$ of the electrolytic capacitor assembly. Based on this consideration, a power density in the range of $6^{-9} \mathrm{~kW} / \mathrm{dm}^{3}$ $\left(100-150 \mathrm{~W} / \mathrm{in}^{3}\right)$ is reasonable for a cost-constraint industrial inverter implementation in accordance with the GLBC specifications.

\section{Conclusion \& OutlooK}

In this paper the experimental results of two GaN-based converter concepts in accordance with the Google Little Box challenge (GLBC) specifications were presented. The first realized inverter, a 2-level H-bridge based inverter topology with interleaved bridge-legs, Triangular Current Mode (TCM) modulation with variable switching frequency in the range of $250 \mathrm{kHz}^{-1} \mathrm{MHz}$, and a buck-type Parallel Current Injector (PCI) power buffer, achieved a power density of $8.18 \mathrm{~kW} / \mathrm{dm}^{3}$ $\left(134 \mathrm{~W} / \mathrm{in}^{3}\right)$, a nominal efficiency of $96.4 \%$ and a California Energy Commission (CEC) weighted efficiency of 95.07\%. This inverter, termed Little Box 1.0 (LB 1.0), was presented at the GLBC finals and was ranked among the top 10 out of $100+$ contestants. The second realized hardware prototype (Little Box 2.0, LB 2.0) brings together all research findings and lessons learned during and after the GLBC and is composed of a 2-level DC/ $|\mathrm{AC}|$ buck-stage operated with constant $140 \mathrm{kHz}$ PWM and a subsequent $\mid \mathrm{AC} / \mathrm{AC} \mathrm{H}$-bridge unfolder. Because of the inherently generated low-frequency Common Mode (CM) voltage, this topology was not considered initially but became a truly viable option once the specified ground current limit was relaxed from $5 \mathrm{~mA}$ to $50 \mathrm{~mA}$ at a very late point during the competition. The LB 2.0, is also equipped with a buck-type PCI buffer but is realized with a more efficient buffer capacitor technology and exhibits a staggering power density of $14.8 \mathrm{~kW} / \mathrm{dm}^{3}\left(243 \mathrm{~W} / \mathrm{in}^{3}\right)$, a nominal efficiency of $97.2 \%$ and a CEC weighted efficiency of $96.1 \%$. Compared to the other LBC finalists as reported in [30], [31], the LB 2.0 inverter therefore sets a new experimentally supported benchmark with respect to power density for a single-phase inverter in accordance with the GLBC specifications.

Given the super-fast switching characteristics of GaN power transistors without reverse recovery, strictly ensuring ZVS throughout the entire mains period is not necessary. In fact, the resulting high RMS current due to TCM bridge-leg control causes high conduction losses which potentially outweighs hard turn-on losses typically occurring around the peak value of the AC current when operated with conventional, constantfrequency PWM but with a comparably large current ripple.

To further improve power density, low-loss ceramic capacitors which tolerate a large AC ripple and feature a very high energy density are needed. This ceramic capacitor technology also must facilitate the implementation of large capacitor blocks in the range of 100-200 $\mu \mathrm{F}$. Equally important is the availability of high-frequency low-loss magnetic materials with high saturation flux density to implement compact, lowloss inductors.

It was demonstrated that with a 2-level bridge-leg implementation of the $\mathrm{DC} /|\mathrm{AC}|$ buck-stage based inverter topology (LB 2.0 ) a power density of $250 \mathrm{~W} / \mathrm{in}^{3}$ is in reach. Based on the current available technologies, a further improvement of power density can be achieved with a flying capacitor multi-level approach to shrink the size of the bridge-leg filter inductors and the EMI filter. As it becomes evident from the work in [25], [26], [32], the volume contribution of providing voltage and signal isolation to the individual switching cells of the flying capacitor converter is, besides the performance limitation imposed by the ceramic capacitors, one of the current barriers for achieving a higher power density. With advanced packaging and a higher level of integration it would not come at a surprise to see power density values in the range of 250-300 W/in ${ }^{3}$ for this type of converter in the very near future. Another strategy to improve the power density is to more tightly incorporate the power buffer into the operation of the inverter as presented in [33], [34], because this would allow to eliminate the bridge-leg filter inductor employed in the PCI buffer. This approach is subject of current research and would potentially allow to improve the power density of the LB 2.0 approach by up to $10 \%-15 \%$.

\section{ACKNOWLEDGEMENT}

The highest level of appreciation goes to G. Deboy from Infineon Austria AG for his active support during the Google Little Box Challenge and for providing a very generous quantity of Infineon CoolGaN transistors used in the LB 1.0 and LB 2.0 hardware prototypes. The authors would also like to express their sincere gratitude to all other team members from ETH Zurich, Fraunhofer IZM, and Fraza involved in the design and construction of the final LB 1.0 prototype.

\section{REFERENCES}

[1] D. Neumayr, D. Bortis, and J. W. Kolar, "The essence of the GOOGLE little box challenge - part A: Key design challenges \& solutions," in CPSS Transactions on Power Electronics and Applications, vol. 5, no. 2, pp. 158-179, Jun. 2020.

[2] Google, "Detailed inverter specifications, testing procedure, and technical approach and testing application requirements for the little box challenge," Google, Tech. Rep., 2015. [Online]. Available: https://www. littleboxchallenge.com

[3] D. Bortis, D. Neumayr, O. Knecht, and J. W. Kolar, "Comprehensive evaluation of GaN GIT in low- and high-frequency bridge leg applications," in Proceedings of 2016 IEEE 8th International Power Electronics and Motion Control Conference, Hefei, China, 2016, pp. 21-30. 
[4] C. P. Henze, H. C. Martin, and D. W. Parsley, "Zero-voltage switching in high frequency power converters using pulse width modulation," in Proceedings of APEC' 88 Third Annual IEEE Applied Power Electronics Conference and Exposition, New Orleans, LA, USA, 1988, pp. 33-40.

[5] C. Marxgut, F. Krismer, D. Bortis, and J. W. Kolar, "Ultraflat interleaved triangular current mode (TCM) single-phase PFC rectifier," in IEEE Transaction on Power Electronics, vol. 29, no. 2, pp. 873-882, Feb. 2014.

[6] R. Wang, F. Wang, D. Boroyevich, R. Burgos, R. Lai, P. Ning, and K. Rajashekara, "A high power density single-phase PWM rectifier with active ripple energy storage," in IEEE Transactions on Power Electronics, vol. 26, no. 5, pp. 1430-1443, Jun. 2011.

[7] Y. Tang, F. Blaabjerg, L. Poh Chiang, J. Chi, and P. Wang, "Decoupling of fluctuating power in single-phase systems through a symmetrical halfbridge circuit," in IEEE Transactions on Power Electronics, vol. 30, no. 4, pp. 1855-1865, Apr. 2015.

[8] A. C. Kyritsis, N. P. Papanikolaou, and E. C. Tatakis, "A novel parallel active filter for current pulsation smoothing on single stage grid-connected AC-PV modules," in Proceedings of 2007 European Conference on Power Electronics and Applications, Aalborg, Denmark, 2007, pp. 1-10.

[9] D. Neumayr, D. Bortis, and J.W. Kolar, "Ultra-compact power pulsation buffer for single-phase DC/AC converter systems," in Proceedings of the 8th International Power Electronics and Motion Control Conference(ECCE Asia / IPEMC), Hefei, China, 2016, pp. 2732-2741.

[10] C. B. Barth, I. Moon, Y. Lei, S. Qin, C. N. Robert, and P. Podgurski, "Experimental evaluation of capacitors for power buffering in singlephase power converters," in Proceedings of 2015 IEEE Energy Conversion Congress and Exposition (ECCE), Montreal, QC, Canada, 2015, pp. 6269-6276.

[11] D. Neumayr, D. Bortis, J. W. Kolar, and J. Konrad, "Comprehensive large-signal performance analysis of ceramic capacitors for power pulsation buffers," in Proceedings of 2016 IEEE 17th Workshop on Control and Modeling for Power Electronics (COMPEL), Trondheim, Norway, 2016, pp. 1-8.

[12] C. B. Barth, T. Foulkes, I. Moon, Y. Lei, S. Qin, C. N. Robert, P. Podgurski, "Experimental evaluation of capacitors for power buffering in single-phase power converters," in IEEE Transactions on Power Electronics, vol. 34, no. 8, pp. 7887-7899, Aug. 2019.

[13] M. Kasper, R. M. Burkart, G. Deboy, and J. W. Kolar, "ZVS of power MOSFETs revisited," in IEEE Transaction on Industrial Electronics, vol. 31, no. 12, pp. 8063-8067, Dec. 2016.

[14] T. Tanaka, T. Ueda, and D. Ueda, "Highly efficient GaN power transistors and integrated circuits with high breakdown voltages," in Proceedings of 2010 10th IEEE International Conference on Solid-State and Integrated Circuit Technology, Shanghai, China, 2010, pp. 1315-1318.

[15] D. Bortis, O. Knecht, and J. W. Kolar, "Vorrichtung zur pulszeitunabh ängigen sicheren Ansteuerung von Leistungstransistoren (in German)," Switzerland Patent CH711 457(A2), Feb. 2017.

[16] F. Zajc, "Multi gap inductor core, multi gap inductor, transformer and corresponding manufacturing method and winding," European Union Patent Application EP 2528069 A1, Nov. 2012.

[17] "Flat band winding for an inductor core," U.S. Patent US2 012299 681(A1), Nov. 2012.

[18] C. R. Sullivan, H. Bouayad, and Y. Song, "Inductor design for low loss with dual foil windings and quasi-distributed gap," in Proceedings of 2013 IEEE Energy Conversion Congress and Exposition, Denver, CO, USA, 2013, pp. 3693-3699.

[19] C. R. Sullivan, "Layered foil as an alternative to litz wire: Multiple methods for equal current sharing among layers," in Proceedings of 2014 IEEE 15th Workshop on Control and Modeling for Power Electronics (COMPEL), Santander, Spain, 2014, pp. 1-7.

[20] D. Neumayr, D. Bortis, J. W. Kolar, S. Hoffmann, and E. Hoene, "Origin and quantification of increased core loss in MnZn ferrite plates of a multi-gap inductor," in CPSS Transactions on Power Electronics and Applications, vol. 4, no. 1, pp. 72-93, Mar. 2019.

[21] D. Zmood and D. Holmes, "Stationary frame current regulation of PWM inverters with zero steady-state error," in IEEE Transacitons on Power
Electronics, vol. 18, no. 3, pp. 814-822, May 2003.

[22] J. Biela, D. Hassler, J. Miniböck, and J. W. Kolar, "Optimal design of a $5 \mathrm{~kW} / \mathrm{dm}^{3} / 98.3 \%$ efficient TCM resonant transition single-phase PFC rectifier," in Proceedings of the 2010 International Power Electronics Conference, Sapporo, Japan, 2010, pp. 1709-1716.

[23] R. W. Erickson and A. P. Rogers, "A microinverter for buildingintegrated photovoltaics," in Proceedings of 2009 24th Annual IEEE Applied Power Electronics Conference and Exposition, Washington, DC, USA, 2009, pp. 911-917.

[24] M. Guacci, M. Heller, D. Neumayr, D. Bortis, J. W. Kolar, G. Deboy, C. Ostermaier, and O. Häberlen, "On the origin of the coss-losses in softswitching GaN-on-Si power HEMTs," in IEEE Journal of Emerging and Selected Topics in Power Electronics, vol. 7, no. 2, pp. 679-694, Jun. 2019.

[25] Y. Lei, C. Barth, S. Qin, W. Liu, I. Moon, A. Stillwell, D. Chou, T. Foulkes, Z. Ye, Z. Liao, C. N. Robert, and P. Podgurski, "A 2 kW, singlephase, 7-Level, GaN inverter with an active energy buffer achieving 216 W/in ${ }^{3}$ power density and $97.6 \%$ peak efficiency," in Proceedings of 2016 IEEE Applied Power Electronics Conference and Exposition (APEC), Long Beach, CA, USA, 2016, pp. 1512-1519.

[26] Y. Lei, C. Barth, S. Qin, W. Liu, I. Moon, A. Stillwell, D. Chou, T. Foulkes, Z. Ye, Z. Liao, C. N. Robert, and P. Podgurski, "A 2-kW singlephase seven-level flying capacitor multilevel inverter with an active energy buffer," in IEEE Transactions on Power Electronics, vol. 32, no. 11, pp. 8570-8581, Nov. 2017.

[27] S. Qin, Y. Lei, C. Barth, W. Liu, C. N. Robert, and P. Podgurski, "Architecture and control of a high energy density buffer for power pulsation decoupling in grid-interfaced applications," in Proceedings of 2015 IEEE 16th Workshop on Control Modeling for Power Electronics (COMPEL), Vancouver, BC, Canada, 2015, pp. 1-8.

[28] R. Ghosh, M. Wang, S. Mudiyula, U. Mhaskar, R. Mitova, D. Reilly, and D. Klikic, "Industrial approach to design a 2-kVA inverter for google little box challenge," in IEEE Transacitons on Industrial Electronics, vol. 65, no. 7, pp. 5539-5549, Dec. 2017.

[29] C. Buttay, C. Martin, F. Morel, R. Caillaud, J. Le Leslé, R. Mrad, N. Degrenne, and S. Mollov, "Application of the PCB-embedding technology in power electronics - State of the art and proposed development," in Proceedings of 2018 Second International Symposium on 3D Power Electronics Integration and Manufacturing (3D-PEIM), College Park, MD, USA, 2018, pp. 1-10.

[30] K. A. Kim, Y. Liu, M. Chen, and H. Chiu, "Opening the box: Survey of high power density inverter techniques from the little box challenge," in CPSS Transactions on Power Electronics and Applications, vol. 2, no. 2, pp. 131-139, Jun. 2017.

[31] C. W. Halsted and M. D. Manjrekar, "A critique of little box challenge inverter designs: Breaking from traditional design tradeoffs," in IEEE Power Electronics Magazine, vol. 5, no. 4, pp. 52-60, Dec. 2018.

[32] P. Papamanolis, D. Neumayr, and J. W. Kolar, "Behavior of the flying capacitor converter under critical operating conditions," in Proceedings of 2017 IEEE 26th International Symposium on Industrial Electronics (ISIE), Edinburgh, UK, 2017, pp. 628-635.

[33] M. Haider, D. Bortis, J. W. Kolar, and Y. Ono, "Novel single-phase Buck+Boost PFC rectifier with integrated series power pulsation buffer," in Proceedings of the 2019 10th International Conference on Power Electronics (ICPE 2019-ECCE Asia), Busan, Korea (South), 2019, pp. $1-10$.

[34] J. A. Cobos, R. Ramos, D. Serrano, J. Oliver, and P. Alou, "Energybuffered single-phase inverter operating in the fundamental limit of indirect power," in Proceedings of 2018 IEEE Energy Conversion Congress and Exposition (ECCE), Portland, OR, USA, 2018, pp. 63566363.

The list of references only includes the literature cited in part B of the paper at hand. Please see part A for further references. 


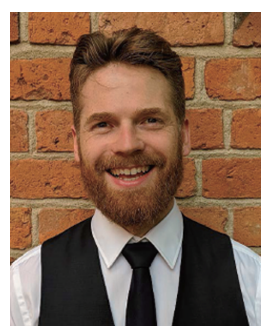

Dominik Neumayr received the M.Sc. and Ph.D. degree in electrical engineering from the Swiss Federal Institute of Technology (ETH) Zurich, Switzerland, in 2015 and 2019, respectively. Between 2008 and 2010, he was with the Center for Advanced Power Systems(CAPS) at Florida State University working on Power/Controller Hardware-in-the-Loop simulations and control systems design for PEBBbased converter systems from ABB. Since spring 2015 he is with the Power Electronic Systems(PES) Laboratory, ETH Zurich. His research focuses on the design, implementation and control of ultracompact and high efficiency converter systems. Mr. Neumayr is currently also acting as technical advisor for rural electrification and off-grid solar power at MPower Ventures.

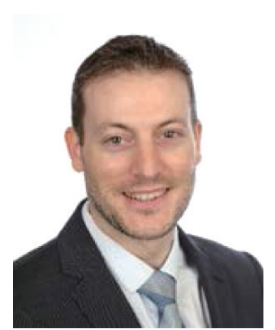

Dominik Bortis received the M.Sc. and Ph.D. degree in electrical engineering from the Swiss Federal Institute of Technology(ETH) Zurich, Switzerland, in 2005 and 2008, respectively. In May 2005, he joined the Power Electronic Systems Labo-ratory(PES), ETH Zurich, as a Ph.D. student. From 2008 to 2011, he has been a Postdoctoral Fellow and from 2011 to 2016 a Research Associate with PES, cosupervising Ph.D. students and leading industry research projects. Since January 2016 Dr. Bortis is heading the research group Advanced Mechatronic Systems at PES, which concentrates on ultra-high speed motors, magnetic bearings and bearingless drives, new linear-rotary actuator and machine concepts with integrated power electronics. In this context, multi-objective optimizations concerning weight/volume/efficiency/ costs, the analysis of interactions of power electronics and electric machines, and EMI are given special attention. Targeted applications include advanced industry automation and manufacturing, e.g. highly dynamic and precise positioning systems, medical and pharmaceutical systems, e.g. ultra-high purity and blood pumps, and future mobility concepts, including motors and actuators for hybrid and electric vehicles, more electric aircraft and satellites. Dr. Bortis has published more than 90 scientific papers in international journals and conference proceedings. He has filed 32 patents and has received 6 IEEE Conference Prize Paper Awards.

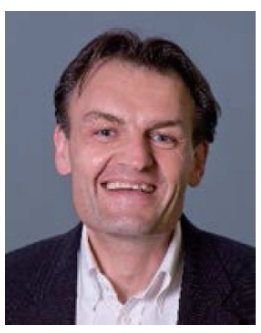

Johann Walter Kolar received his M.Sc. and Ph.D. degree (summa cum laude/promotio sub auspiciis praesidentis rei publicae) from the University of Technology Vienna, Austria, in 1997 and 1999, respectively. Since 1984, he has been working as an independent researcher and international consultant in close collaboration with the University of Technology Vienna, in the fields of power electronics, industrial electronics and high performance drive systems. He has proposed numerous novel PWM converter topologies, modulation and control concepts and has supervised 70+ Ph.D. students. He has published $880+$ scientific papers in international journals and conference proceedings, 4 book chapters, and has filed 190+ patents. The focus of his current research is on ultra-compact and ultra-efficient $\mathrm{SiC}$ and $\mathrm{GaN}$ converter systems, solid-state transformers, advanced variable speed three-phase motor drives, integrated modular motor drives, ultra-high speed motors, bearingless motors/ actuators, and design automation in power electronics/mechatronics. Dr. Kolar has received 31 IEEE Transactions and Conference Prize Paper Awards, the 2014 IEEE Middlebrook Award, the 2016 IEEE William E. Newell Power Electronics Award, the 2016 IEEE PEMC Council Award and two ETH Zurich Golden Owl Awards for excellence in teaching. He initiated and/or is the founder of four ETH Spin-off companies. He is a member of the steering committees of several leading international conferences in the field and has served from 2001 through 2013 as an associate editor of the IEEE Transactions on Power Electronics. Since 2002 he also is an associate editor of the Journal of Power Electronics of the Korean Institute of Power Electronics and a member of the Editorial Advisory Board of the IEEJ Transactions on Electrical and Electronic Engineering. 